\title{
Multi-targeted directed ligands for Alzheimer's disease: design of novel lead coumarin conjugates
}

\author{
B. P. Repsold, S. F. Malan, J. Joubert and D. W. Oliver
}

\begin{abstract}
Alzheimer's Disease (AD) is a neurodegenerative disease characterized by central nervous system insults with progressive cognitive (memory, attention) and non-cognitive (anxiety, depression) impairments. Pathophysiological events affect predominantly cholinergic neuronal loss and dysfunctions of the dopaminergic system. The aim of the current study was to design multi-targeted directed lead structures based on the coumarin scaffold with inhibitory properties at two key enzymes in disease relevant systems, i.e. acetylcholinesterase (AChE) and monoamine oxidase B (MAO-B). Conventional and microwave synthetic methods were utilized to synthesize coumarin scaffoldbased novel morpholino, piperidino, thiophene and erucic acid conjugates. Biological assays indicated that the coumarinmorpholine ether conjugate BPR 10 was the most potent hMAO-B inhibitor. The coumarinpiperidine conjugates BPR 13 and BPR 12 were the most potent inhibitors of eeAChE at 100 $\mu \mathrm{M}$ and $1 \mu \mathrm{M}$, respectively. Molecular modelling studies were conducted with Accelrys $\AA$ Discovery Studio® V3.1.1 utilising the published hMAO-B (2V61) and hAChE (4EY7) crystal structures. Compound BPR 10 occupies both the entrance and substrate cavities of the active site of MAO-B. BPR 13 resides in both the peripheral anionic site (PAS) and the catalytic anionic site (CAS) of hAChE. This study demonstrated that the coumarin scaffold serves as a promising pharmacophore for MTDLs design.
\end{abstract}

\section{Introduction}

Alzheimer's disease (AD) is categorized as the most dominant form of neurodegenerative disease leading to dementia. Common symptoms include a decline in cognition (learning and memory) but it also affects non-cognitive abilities (e.g. anxiety, depression, apathy and psychosis). A 2015 survey revealed that, worldwide, 46.8 million people are living with dementia [1]. Statistics predict that, worldwide in 2050, an individual could develop AD every three seconds [2]. Pathologically AD progresses to affect limbic structures, subcortical nuclei and cortical regions, causing disturbance of multiple neurotransmitter systems. The most dominant neuronal loss is in the cholinergic system [3,4], in which the cholinergic neurons and the number of nicotinic acetylcholine receptors (nACHRs) declines in the hippocampus and cortex [5,6]. This hippocampal atrophy can spread to the amygdala [7]. Non-cognitive behavioural and neuropsychiatric symptoms often accompany $\mathrm{AD}[8]$ and usually arise from the dysfunction of the serotonergic and dopaminergic systems $[8,9]$. The characteristics of $\mathrm{AD}$ include the presence of extracellular plaques containing the beta-amyloid protein $\left(\mathrm{A} \beta_{42}\right)$ and intracellular neurofibrillary tangles 
(NFTs) of hyperphosphorylated tau protein with a further downstream effect of neuronal synapse degeneration [10].

No single 'magic bullet' exists to prevent or cure $\mathrm{AD}$, and current treatments only provide symptomatic relief that doesn't significantly address the underlying neurodegeneration and pathophysiology [11]. Currently approved treatment regimens include three acetylcholinest-erase (AChE) inhibitors (donepezil, rivastigmine and galantamine) and a non-competitive $N$-methyl-Daspartate (NMDA) antagonist (memantine). Usually, this is used in conjunction with a mood stabilizer (since AD isn't limited to cognitive effects), resulting in a 'cocktail' of medication (Figure 1).

Medicinal chemistry research in the past generally focused on highly specific and selective compounds for single target interaction, also known as a one-molecule, one-target paradigm, and proved to be effective for disorders where a single target has been identified. As pharmacogenomics and other biochemical fields progressed, it became evident that the underlying mechanisms of disease are significantly more complex. Additionally, cells can compensate during drug intervention, by amongst others, the existence of parallel pathways [12]. Researchersbegan applying anewstrategybased on therationale that asinglecompound mightbe able to interact with multiple targets for the treatment of neurodegenerative diseases [13]. This approach is known as multi-target-directed ligands (MTDLs) (Figure 2).

The aim of the current investigation was to design multi-target directed lead structures based on the coumarin scaffold. The purpose was to design compounds that inhibit both the cholinergic and dopaminergic systems by inhibiting the two key enzymes, i.e. acetyl-cholinesterase (AChE) and monoamine oxidase B (MAO-B). MAO-B inhibition has been closely associated with AD pathology, including the accompanied neuropsychiatric symptoms [8], anti-oxidant effects/oxidative stress generated by radical oxygen species (ROS) [13-15], A $\beta$ aggregation inhibition [13,16], inhibition of $\beta$ secretase [16], metal chelating activity [14,17] and the formation of tau aggregates [18]. The current investigation employed conventional and microwave-assisted synthetic methods; molecular modelling studies using the CDOCKER ${ }^{\circledR}$ module of Accelrys ${ }^{\circledR}$ Discovery Studio ${ }^{\circledR}$ V3.1.1 software (Dassault Systemes, BIOVIA Corp., San Diego, CA) utilizing the published hMAO-B (2V61) and hAChE (4EY7) crystal structures [19-22]; and biological evaluation of acetylcholinesterase (AChE) and monoamine oxidase B (MAO-B) inhibition by the newly synthesized compounds. 


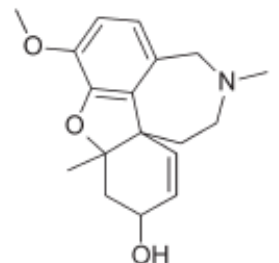

Galantamine

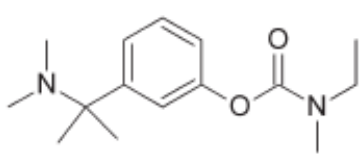

Rivastigmine
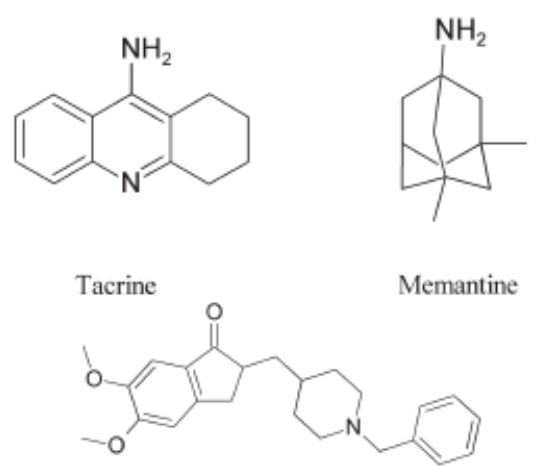

Donepezil

Figure 1. Current treatment medications for AD.

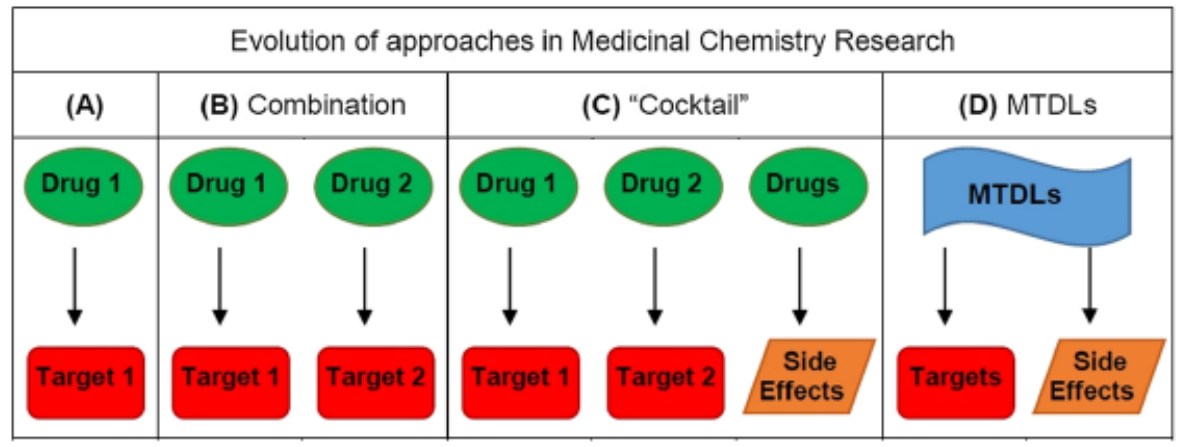

Figure 2. The evolution of approaches in medicinal chemistry research. (a) Specific drugs for specific target; (b) specialized drugs for relevant targets; (c) the 'cocktail' effect, when numerous drugs are dispensed for treatment of targets as well as side effects; (d) a new drug entity that addresses both the underlying pathology as well as side effects (adapted from [12]).

\section{Design of conjugated coumarin scaffolds for Alzheimer's disease}

The current study allows for the design of structures of coumarin conjugated with selected structural moieties, i.e. piperidine, morpholine, thiophene and erucic acid, which could potentially act as lead pharmacophoric MTDLs. The coumarin scaffolds (Figure 3) allow for conjugates with inhibition of $\mathrm{AChE}$ [23] characterized by dual interaction with both the active and peripheral sites of AChE. These compounds therefore also have the advantage of inhibiting AChEinduced $A \beta$ aggregation [24]. Coumarin structures have shown neuroprotective effects related to the interaction with $\beta$-secretase enzyme (BACE-1) [24], NMDA [25] and GABA-A receptors [26]. Additionally, coumarin's other properties include protection against $A \beta$ induced neuronal apoptosis, the regulation of L-type voltage-dependent calcium channels (VDCCs), which in turn causes a decrease of intracellular $\mathrm{Ca}^{2+}$ concentration [27], and counteracting oxidative stress induced by $\mathrm{H}_{2} \mathrm{O}_{2}$ [12-14,28]. 


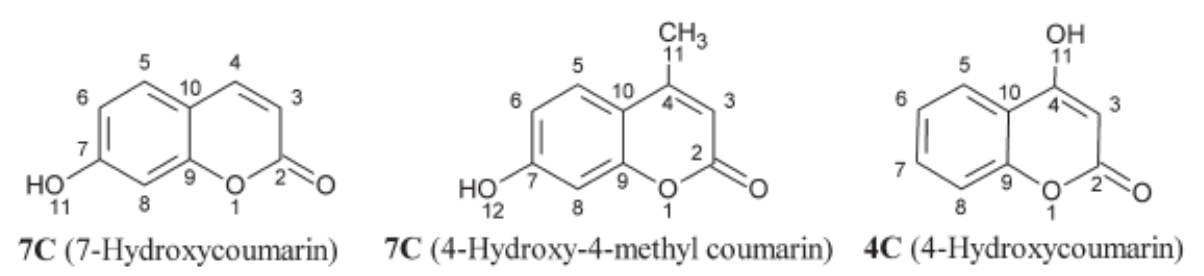

Figure 3. Coumarin pharmacophores employed in this study.

\section{Conjugated moieties (piperidine, morpholine, thiophene and erucic acid)}

It has been found that piperdine (Figure 4) hybrids act as $\gamma$-secretase modulators, with high in vitro and in vivo anti-A $\beta_{42}$ potency [29-32], $A \beta_{42}$ aggregation inhibitors, AChE-induced $A \beta$ aggregation [33], moderate to good AChE inhibitory activity [33,34] and positive metal-chelating ability [35]. Morpholine (Figure 4) contributes to decreased metal-induced ( $\mathrm{Fe}$ and $\mathrm{Cu}$ ) $\mathrm{A} \beta$ aggregation [36]; reduces A $\beta$ toxicity, e.g. compound [(G3)-Mor] [37]; acts as an muscarinic receptor 1 agonist; enhances memory function in Alzheimer's type dementia animal models; and modulates APP secretion [38,39]. It became evident that various thiophene (Figure 4) conjugates were potential AChE inhibitors [40-42]. Erucic acid (Figure 4) is a monounsaturated omega-9 fatty acid present in wallflower seed, rapeseed oil and canola (20-54\%), and in mustard oil (42\%). Erucic acid deficiency was detected in the early phase of neurodegeneration, and this strongly supported its use as a supplement to counteract AD progression [43]. Rapeseed proved to offer protection against $\mathrm{A} \beta$-mediated cell death [44].

The coumarin structure with substitution options at positions 4, 7 and both (Figure 3) allows for unique chemical modifications resulting in novel structures that could serve as novel leads for further design and discovery research focussing on the MTDL approach for AD. Previous studies conducted with these substituted coumarins proved to be beneficial and provide valuable insight or clarify the rationale substitution preference into the structure activity relations (SAR) versus multiple target potency.

\section{Chemistry}

\section{Materialsandinstrumentation}

\section{Materials}

Starting materials were obtained from Sigma-Aldrich and Merck (Merck (Pty) Ltd, Modderfontein, Gauteng, Republic of South Africa (RSA)) and was used without further purification or converted to intermediate (more reactive) derivatives. All solvents used were anhydrous. Drying after extraction of the organic fractions was achieved using magnesium sulphate $\left(\mathrm{MgSO}_{4}\right)$.

\section{Thin layer chromatography}

Thin layer chromatography (TLC) was carried out to determine the completeness of the reaction. Silica gel 60 sheets (Merck) containing $\mathrm{UV}_{254}$ fluorescent indicator were employed with the appropriate mobile phase for each reaction. The developed TLC sheets were observed under UV at a wavelength of $354 \mathrm{~nm}$ and the $\mathrm{R} f$ values noted.

\section{http://repository.uwc.ac.za}




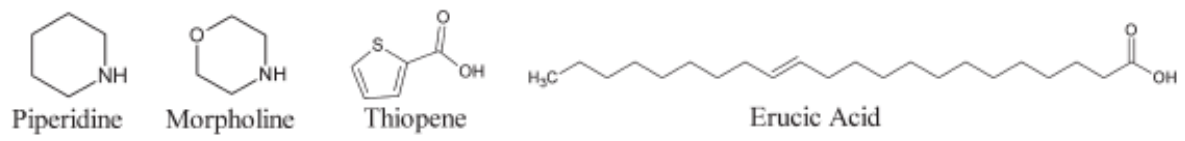

Figure 4. Structural moieties utilized for conjugation to coumarin.

\section{Melting points (Mp)}

A Büchi B- $545^{\circledR}$ melting point apparatus (Labotec (PTY) Ltd (Büchi SA), Gauteng, RSA) was used to measure the Mp of all synthesised compounds.

\section{Mass spectra (MS)}

High resolution mass spectra (HRMS) and nominal mass spectra (MS) were obtained with a Bruker micrOTOF-Q II ${ }^{\circledR}$ mass spectrometer (Bruker South Africa (Pty) LTD, Sandton, Gauteng, RSA) in atmospheric-pressure chemical ionization (APCI) mode.

\section{Nuclear magnetic resonance (NMR)}

A Bruker Avance III $600{ }^{\circledR}$ spectrometer, at frequencies of $600 \mathrm{MHz}$ and $150 \mathrm{MHz}$, was used to record proton $\left({ }^{1} \mathrm{H}\right)$ and carbon $\left({ }^{13} \mathrm{C}\right)$ nuclear magnetic resonance (NMR) spectra, respectively. NMR experiments were conducted in $\mathrm{CDCl}_{3}$ and the chemical shifts reported in parts per million $(\delta)$ downfield from tetramethylsilane (TMS). Spin multiplicities are given as s (singlet), bs (broad singlet), d (doublet), dd (doublet of doublets), t (triplet), q (quartet) or $\mathrm{m}$ (multiplet). The coupling constants $(J)$ of the molecules' atoms involved are given in $\mathrm{Hz}$.

\section{Microwave-assisted synthesis}

ACEM Discovery ${ }^{\circledR}$ microwave reactor (Mintek, Randburg, Gauteng, RSA) fitted with a cooler, a 100 $\mathrm{mL}$ flask with the suitable volume of solvent together with the adapted cooling system (as discussed below) was implemented (Figure 5). The advantages of microwave-assisted synthesis are:

Extreme reaction rate acceleration that is associated with uncomplicated purification/isolation; Enhanced physicochemical properties; Yield improvement; Generation of new 'potential products' and Financial benefits, e.g. less solvent(s) is used, absence of expensive catalyst, etc. [45].

Thedisadvantages of microwave-assisted synthesis are:

Limitation of solvent (because of the microwave-induced thermal effect). Solvents with a low boiling point are excited much more easily, which makes cooling/reflux much more uncontrollable, therefore we only choose solvents with a boiling point of $60^{\circ} \mathrm{C}$ and above; The generation of new product(s)/byproduct(s) can complicate the isolation of the desired products; Temperature regulation (sometimes cooling with nitrogen is required); and High investment costs [46]. 


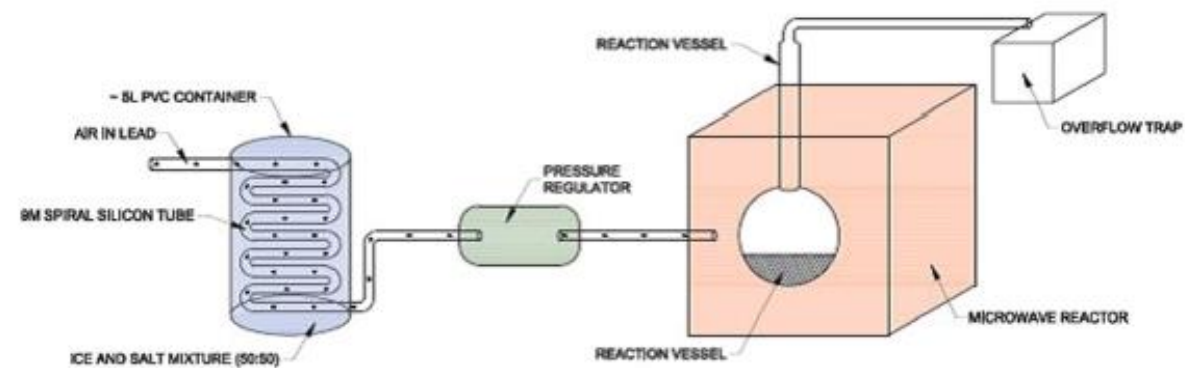

Figure 5 . Schematic outlay of the modified microwave reactor used in this study.

\section{Syntheticroutes}

\section{Erucic acid-coumarin ester conjugates}

Erucic acid was firstly converted to its corresponding acyl chloride by means of oxalyl chloride in dry dichloromethane (DCM) to provide a more efficient nucleophilic attack on the respective coumarins (Scheme1).

\section{Coumarinetherdimers}

Syntheses of dimers were done according to the classical Williamson etherification where the $7 \mathrm{C}$, $4 \mathrm{MC}$ and $4 \mathrm{C}$ (Scheme 2) served as nucleophiles and displaced the appropriate halide (1,3-dibromopropane and 1,4-dibromo-ethane) via the $\mathrm{Sn} 2$ reaction to produce the corresponding coumarin dimer. A basic salt $\left(\mathrm{K}_{2} \mathrm{CO}_{3}\right)$ and a spatula point of $\mathrm{KI}$ were used as suitable 'catalysts' in acetonitrile (Scheme 2).

\section{Coumarin-thiophene ester conjugates}

Activating the thiophene acid in situ using N,N'-carbonyldiimidazole (CDI) in dry DCM provides the appropriate conditions for nucleophillic attack by $7 \mathrm{C}$ to produce the coumarin-thiophene conjugate (Scheme 3).

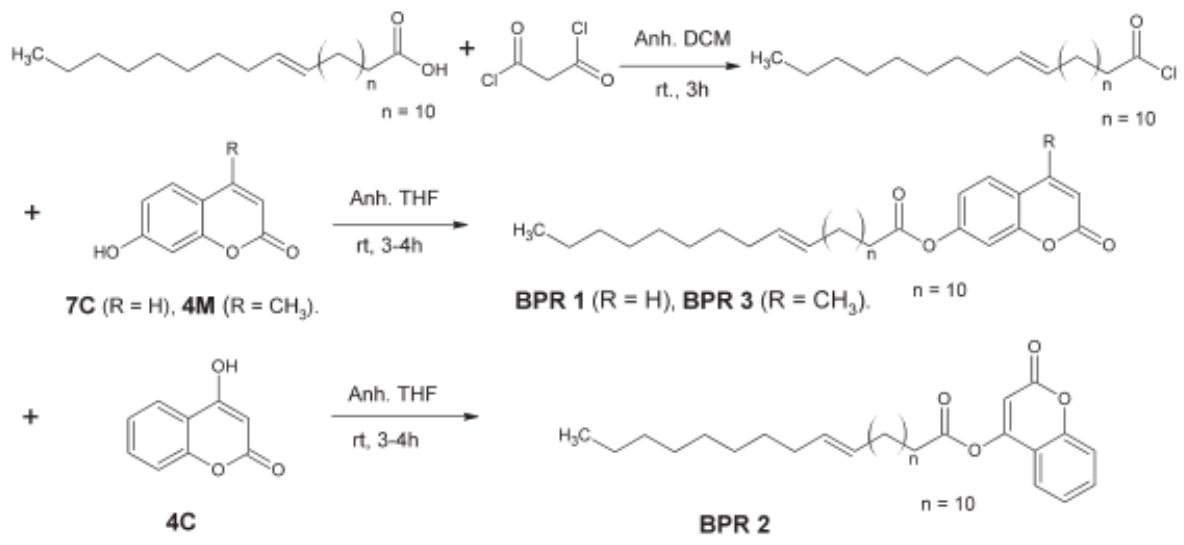

Scheme 1. The synthesis of erucic acid-coumarin conjugates (BPR1-BPR3). 


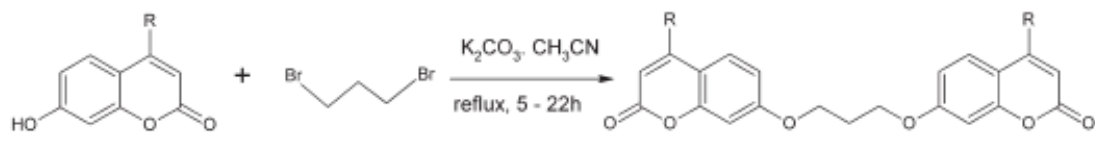

$7 \mathrm{C}(\mathrm{R}=\mathrm{H}), 4 \mathrm{M}\left(\mathrm{R}=\mathrm{CH}_{3}\right)$

BPR $6(\mathrm{R}=\mathrm{H})$, BPR $7\left(\mathrm{R}=\mathrm{CH}_{3}\right)$.

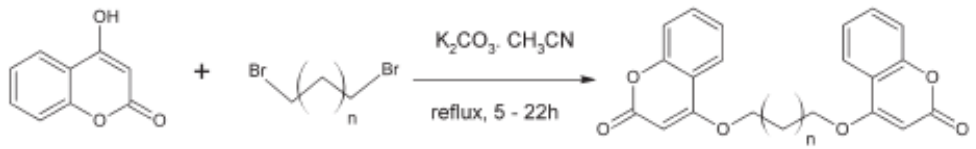

4C $\quad n=2, n=1 \quad$ BPR $4(n=2)$, BPR $5(n=1)$

Scheme 2. Synthesis of coumarin dimer derivatives (BPR 4-BPR 7).

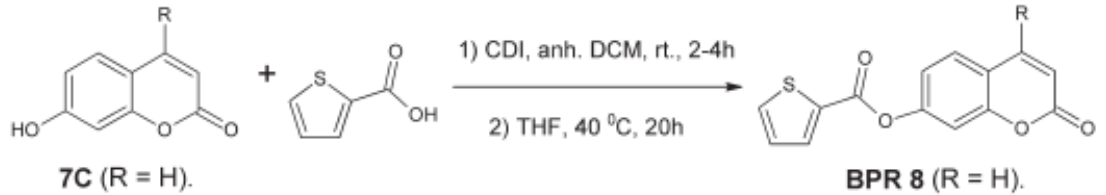

Scheme 3. Synthesis of coumarin-thiophene conjugate (BPR8).
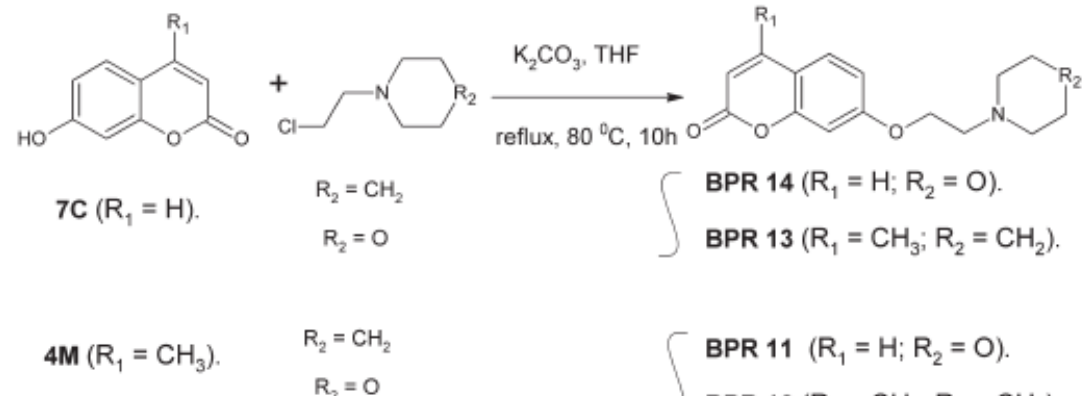

BPR $11\left(R_{1}=H ; R_{2}=0\right)$

BPR $12\left(\mathrm{R}_{1}=\mathrm{CH}_{3} ; \mathrm{R}_{2}=\mathrm{CH}_{2}\right)$.<smiles>O=c1cc(O)c2ccccc2o1</smiles>

$4 \mathrm{C}$

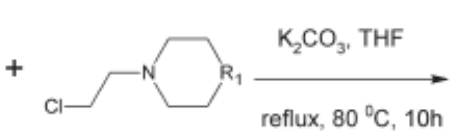

$\mathrm{R}_{1}=\mathrm{CH}_{2}$

$R_{1}=0$

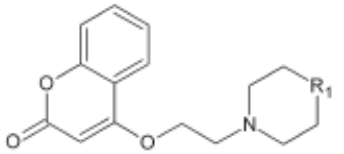

BPR $10\left(R_{1}=H ; R_{2}=0\right)$.

BPR $9\left(\mathrm{R}_{1}=\mathrm{CH}_{3} ; \mathrm{R}_{2}=\mathrm{CH}_{2}\right)$.

Scheme 4. Synthetic route of coumarin derivates conjugated with piperidine and morpholine (BPR 9-BPR 14).

\section{Coumarin-piperidine ethersand coumarin-morpholine etherconjugates}

Etherfication of $7 \mathrm{C}, 4 \mathrm{M}$ and $4 \mathrm{C}$ (Figure 3) with chloro-ethane piperidine and chloro-ethane morpholine through conventional heating produced the respective coumarin conjugates (Scheme 4). 


\section{Synthetic methodology and compound characteristics}

Coumarin-erucic ester conjugates (BPR 1-BPR 3)

A mixture of $3.36 \mathrm{mmol}, 1139 \mathrm{mg}$, erucic acid and $3.36 \mathrm{mmol}, 427 \mathrm{mg}$ oxacyl chloride was suspended in $30 \mathrm{~mL}$ dry DCM and stirred for $2 \mathrm{~h}$ at room temperature (rt). The reaction turned yellowish and the corresponding erucyl chloride was formed. The excess oxacyl chloride as well as the DCM were removed under vacuum $\left(60^{\circ} \mathrm{C}\right)$. The remaining erucyl chloride was suspended in 30 $\mathrm{mL}$ tetrahydrofuran (THF) and, on an ice bath, $3.70 \mathrm{mmol}, 600 \mathrm{mg}, 7 \mathrm{C}$ and $7.40 \mathrm{mmol}, 1022 \mathrm{mg}, 2.2$ mol eqv. $\mathrm{K}_{2} \mathrm{CO}_{3}$ were added. The suspension was stirred for another $3-4 \mathrm{~h}$ at $\mathrm{rt}$, filtered and purified using column chromatography with DCM: cyclohexane (3:1). It was dried, filtered and concentrated to obtain a white wax with a sweet odour (BPR 1). Yield: $860 \mathrm{mg}, 1.78 \mathrm{mmol}, 48.11 \%$.

\section{BPR 1: 2-Oxo-2H-chromen-7-yl-docos-24-enoate}

Rf: 0.491. Mp: 53.8-54.5 ${ }^{\circ} \mathrm{C} .{ }^{1} \mathrm{H}$ NMR (600 MHz, $\left.\mathrm{CDCI}_{3}\right): \delta 7.67\left(\mathrm{~d}, J=9.5 \mathrm{~Hz}, 1 \mathrm{H}, \mathrm{H}_{4}\right) ; 7.46(\mathrm{~d}, J=8.4 \mathrm{~Hz}$, $\left.1 \mathrm{H}, \mathrm{H}_{5}\right) ; 7.08\left(\mathrm{~d}, J=2.1 \mathrm{~Hz}, 1 \mathrm{H}, \mathrm{H}_{6}\right) ; 7.04-6.99\left(\mathrm{~m}, 1 \mathrm{H}, \mathrm{H}_{8}\right) ; 6.37\left(\mathrm{~d}, J=9.5 \mathrm{~Hz}, 1 \mathrm{H}, \mathrm{H}_{3}\right) ; 5.32(\mathrm{t}, J=4.7$ $\mathrm{Hz}, 2 \mathrm{H}, \mathrm{H}_{24,25}$ ); 2.56 (t, $J=7.5 \mathrm{~Hz}, 2 \mathrm{H}, \mathrm{H}_{13}$ ); 1.99 (m, $4 \mathrm{H}, \mathrm{H}_{23,26}$ ); 1.77-1.70 (m, 4 H, $\mathrm{H}_{14}$ ); 1.35-1.19 (m, 28H, H15-22,27-32); 0.85 (t, $J=7.0 \mathrm{~Hz}, 3 \mathrm{H}, \mathrm{H}_{33}$ ). C NMR (151 MHz, $\mathrm{CDCI}_{3}$ ): $8171.61\left(\mathrm{C}_{12}\right) ; 160.35$ $\left(\mathrm{C}_{2}\right) ; 154.68\left(\mathrm{C}_{9}\right) ; 153.29\left(\mathrm{C}_{7}\right) ; 142.84\left(\mathrm{C}_{4}\right) ; 129.91\left(\mathrm{C}_{24}\right) ; 129.85\left(\mathrm{C}_{25}\right) ; 118.43\left(\mathrm{C}_{5}\right) ; 116.56\left(\mathrm{C}_{6}\right) ; 116.00$ $\left(\mathrm{C}_{10}\right) ; 110.43\left(\mathrm{C}_{3}\right) ; 34.32\left(\mathrm{C}_{8}\right) ; 31.89\left(\mathrm{C}_{13}\right) ; 30.92\left(\mathrm{C}_{31}\right) ; 29.75^{-29.05}\left(\mathrm{C}_{15-23,26-29}\right) ; 27.19\left(\mathrm{C}_{30}\right) ; 24.77\left(\mathrm{C}_{14}\right)$; $22.67\left(\mathrm{C}_{32}\right) ; 14.18\left(\mathrm{C}_{33}\right)$. MS: APCI-HRMS $\mathrm{m} / \mathrm{z}$ : calculated for $\mathrm{C}_{31} \mathrm{H}_{46} \mathrm{O}_{4}\left(\mathrm{MH}^{+}\right)$, 483.3469, found 481.3297 .

\section{BPR 2: 2-Oxo-2H-chromen-4-yl docos-24-enoate}

Employing the above-mentioned procedure (Coumarin-erucic ester conjugates (BPR 1-BPR 3)), using $3.70 \mathrm{mmol}$, $600 \mathrm{mg}$, $4 \mathrm{C}$ and $7.40 \mathrm{mmol}, 1022 \mathrm{mg} \mathrm{K}_{2} \mathrm{CO}_{3}$. Purification: column chromatography with DCM:cyclohexane (8:2). A white wax with a sweet odour formed.

Rf: 0.585. Mp: $77-79^{\circ} \mathrm{C} .{ }^{1} \mathrm{H}$ NMR $\left(600 \mathrm{MHz}, \mathrm{CDCl}_{3}\right): \delta 7.60\left(\mathrm{dd}, J=7.9,1.5 \mathrm{~Hz}, 1 \mathrm{H}, \mathrm{H}_{5}\right) ; 7.56$ (m, $\left.1 \mathrm{H}, \mathrm{H}_{7}\right) ; 7.34$ (dd, $\left.J=1,0.6 \mathrm{~Hz}, 1 \mathrm{H}, \mathrm{H}_{6}\right) ; 7.30-7.26$ (m, $\left.1 \mathrm{H}, \mathrm{H}_{8}\right) ; 6.50\left(\mathrm{~s}, 1 \mathrm{H}, \mathrm{H}_{3}\right), 5.33(\mathrm{~m}, 2 \mathrm{H}$, $\mathrm{H}_{26,27}$ ); 2.68 (t, $J=7.5 \mathrm{~Hz}, 2 \mathrm{H}, \mathrm{H}_{13}$ ); 1.77 (m, $4 \mathrm{H}, \mathrm{H}_{25,28}$ ); 1.39-1.17 (m, 28H, $\mathrm{H}_{15-24,29-32}$ ); 0.85 (t, $\left.J=7.0 \mathrm{~Hz},{ }_{3} \mathrm{H}, \mathrm{H}_{33}\right) .{ }^{13 \mathrm{C} \mathrm{NMR}}\left(151 \mathrm{MHz}, \mathrm{CDCI}_{3}\right): \delta 169.53\left(\mathrm{C}_{12}\right) ; 161.50\left(\mathrm{C}_{4}\right) ; 158.34\left(\mathrm{C}_{2}\right) ; 153.65\left(\mathrm{C}_{9}\right)$; $132.71\left(\mathrm{C}_{7}\right) ; 129.92\left(\mathrm{C}_{26}\right) ; 129.83\left(\mathrm{C}_{27}\right) ; 124.26\left(\mathrm{C}_{5}\right) ; 122.66\left(\mathrm{C}_{6}\right) ; 117.09\left(\mathrm{C}_{8}\right) ; 115.53\left(\mathrm{C}_{10}\right) ; 105.06\left(\mathrm{C}_{3}\right)$; $34.55\left(\mathrm{C}_{13}\right) ; 30.92\left(\mathrm{C}_{31}\right) ; 29.75^{-29.16}\left(\mathrm{C}_{15^{-25}}\right)$; $29.00\left(\mathrm{C}_{28}\right) ; 27.19\left(\mathrm{C}_{29}\right) ; 26.89\left(\mathrm{C}_{30}\right) ; 24.65\left(\mathrm{C}_{14}\right)$; $22.67\left(\mathrm{C}_{32}\right)$; $14.10\left(\mathrm{C}_{33}\right)$. MS: APCI-HRMS m/z: calculated for $\mathrm{C}_{31} \mathrm{H}_{46} \mathrm{O}_{4}\left(\mathrm{MH}^{+}\right), 483.3469$, found 481.303. Yield: $710 \mathrm{mg}, 1.47 \mathrm{mmol}, 39.73 \%$.

\section{BPR 3: 4-Methyl-2-oxo-2H-chromen-7-yl docos-25-enoate}

Employing the above-mentioned procedure (Coumarin-erucic ester conjugates (BPR 1-BPR 3)), using $3.40 \mathrm{mmol}$, $600 \mathrm{mg}$, $4 \mathrm{M}$ and $3.78 \mathrm{mmol}, 1023 \mathrm{mg}, \mathrm{K}_{2} \mathrm{CO}_{3}$ were added, stirred for $14 \mathrm{~h}$ at rt. Purification: column chromatography with DCM:cyclohexane (7:1). A white wax with a sweet odour formed.

\section{http://repository.uwc.ac.za}


Rf: 0.528. Mp: $49-51.2^{\circ} \mathrm{C} .{ }^{1} \mathrm{H}$ NMR $\left(600 \mathrm{MHz}, \mathrm{CDCl}_{3}\right): \delta 7.58$ (d, $\left.J=8.6 \mathrm{~Hz}, 1 \mathrm{H}, \mathrm{H}_{5}\right) ; 7.08$ (d, $J$ $\left.=2.2 \mathrm{~Hz}, 1 \mathrm{H}, \mathrm{H}_{6}\right) ; 7.04\left(\mathrm{dd}, J=8.6,2.3 \mathrm{~Hz}, 1 \mathrm{H}, \mathrm{H}_{8}\right) ; 6.24\left(\mathrm{~s}, 1 \mathrm{H}, \mathrm{H}_{3}\right) ; 5.37-5.29\left(\mathrm{~m}, 2 \mathrm{H}, \mathrm{H}_{25,26}\right) ; 2.56(\mathrm{t}, J$ $\left.=7.5 \mathrm{~Hz}, 2 \mathrm{H}, \mathrm{H}_{14}\right) ; 2.41\left(\mathrm{~s}, 3 \mathrm{H}, \mathrm{H}_{11}\right) ; 2.03-1.95\left(\mathrm{~m}, 4 \mathrm{H}, \mathrm{H}_{24,27}\right) ; 1.77-1.70\left(\mathrm{~m}, 2 \mathrm{H}, \mathrm{H}_{15}\right) ; 1.33-1.21(\mathrm{~m}, 28 \mathrm{H}$, $\left.\mathrm{H}_{16-13,28-33}\right) ; 0.85$ (t, $\left.J=7.0 \mathrm{~Hz}, 3 \mathrm{H}, \mathrm{H}_{34}\right) .{ }^{13} \mathrm{C} \mathrm{NMR}\left(151 \mathrm{MHz}, \mathrm{CDCI}_{3}\right): \delta 171.64\left(\mathrm{C}_{13}\right) ; 160.51\left(\mathrm{C}_{2}\right)$; $154.17\left(\mathrm{C}_{7}\right) ; 153.18\left(\mathrm{C}_{9}\right) ; 151.87\left(\mathrm{C}_{4}\right) ; 129.90\left(\mathrm{C}_{25}\right) ; 125.30\left(\mathrm{C}_{26}\right) ; 118.11\left(\mathrm{C}_{5}\right) ; 117.73\left(\mathrm{C}_{6}\right) ; 114.45\left(\mathrm{C}_{10}\right)$; $110.44\left(\mathrm{C}_{3}\right) ; 34.32\left(\mathrm{C}_{8}\right) ; 31.89\left(\mathrm{C}_{14}\right) ; 30.92\left(\mathrm{C}_{32}\right) ; 29.75-27.19\left(\mathrm{C}_{27-31,16-24}\right) ; 24.77\left(\mathrm{C}_{15}\right) ; 22.67\left(\mathrm{C}_{33}\right) ; 18.71$ $\left(\mathrm{C}_{11}\right) ; 14.10\left(\mathrm{C}_{34}\right)$. MS: APCI-HRMS m/z: calculated for $\mathrm{C}_{32} \mathrm{H}_{48} \mathrm{O}_{4}\left(\mathrm{MH}^{+}\right)$, 497.3625, found 497.3558. Yield: $685 \mathrm{mg}, 1.38 \mathrm{mmol}, 40.59 \%$.

\section{Coumarin-ether dimers (BPR 4-BPR 7)}

In a $50 \mathrm{~mL}$ flask, $3.08 \mathrm{mmol}, 500 \mathrm{mg}$, $4 \mathrm{C} ; 6.16 \mathrm{mmol}, 852 \mathrm{mg} \mathrm{K} \mathrm{CO}_{3}$ and $3.08 \mathrm{mmol}, 123 \mathrm{mg}$, NaH (60\% dispersed in oil) were suspended in $6-8 \mathrm{~mL}$ dimethylformamide (DMF). It was stirred for $30 \mathrm{~min}$ at rt, and to this stirred solution $1.54 \mathrm{mmol}, 333 \mathrm{mg}$ 1,4-dibromobutane was slowly added, and it was stirred for a further $20 \mathrm{~h}$ at $50^{\circ} \mathrm{C}$. The mixture was filtered and $40-50 \mathrm{~mL}$ water was added to the suspension until a white precipitate formed. It was washed with cyclohexane to dispose of the excess DMF. Yield: $195 \mathrm{mg}$, $0.52 \mathrm{mmol}, 16.88 \%$.

\section{Alternative method}

In a $50 \mathrm{~mL}$ flask, $3.08 \mathrm{mmol}, 500 \mathrm{mg}, 4 \mathrm{C} ; 6.16 \mathrm{mmol}, 852 \mathrm{mg}, \mathrm{K}_{2} \mathrm{CO}_{3}$ and $3.08 \mathrm{mmol}, 123 \mathrm{mg}$, NaH (60\% dispersed in oil) were suspended in $30 \mathrm{~mL}$ anhydrous acetonitrile and stirred for $30 \mathrm{~min}$ at $\mathrm{rt}$, and $1.54 \mathrm{mmol}, 333 \mathrm{mg}$ 1,4-dibromobutane was slowly added. Using the microwave reactor, the solution was irradiated according the following preferences: open vessel with cooling on; power $200 \mathrm{~W}$; temperature $45^{\circ} \mathrm{C}$; run time 1:30 min; hold time 1:30 min. It was cooled down between sessions and irradiation repeated another three times. The reaction mixture was filtered, concentrated and recrystallized to yield white crystals (BPR 4).

\section{BPR 4: 4,4'-[Butane-1,4-diylbis(oxy)]bis(2H-chromen-2-one)}

Rf: 0.282. Mp: $173.2-175.2^{\circ} \mathrm{C} .{ }^{1} \mathrm{H}$ NMR (600 MHz, CDCl ): $\delta 7.77$ (dd, $J=7.9,1.4 \mathrm{~Hz}, 2 \mathrm{H}, \mathrm{H} 5,21$ ); $7.56-$ 7.49 (m, $2 \mathrm{H}, \mathrm{H}_{7,23}$ ); 7.28-7.20 (m, 4H, H8,24,6,22); 5.66 (s, $2 \mathrm{H}, \mathrm{H}_{3,19}$ ); 4.29 (bs, $2 \mathrm{H}, \mathrm{H}_{12,15}$ ); 2.23 (bs, $\left.2 \mathrm{H}, \mathrm{H}_{13,14}\right) ; 1.63\left(\mathrm{~s}, 2 \mathrm{H}, \mathrm{H}_{2} \mathrm{O} ; \mathrm{CDCI}_{3}\right) .{ }_{13 \mathrm{C}} \mathrm{NMR}\left(151 \mathrm{MHz}, \mathrm{CDCl}_{3}\right): \delta 165.37\left(\mathrm{C}_{4,20}\right) ; 162.77\left(\mathrm{C}_{2,18}\right)$; $153.26\left(\mathrm{C}_{9,25}\right) ; 132.53\left(\mathrm{C}_{7,23}\right) ; 123.90\left(\mathrm{C}_{5,21}\right) ; 122.71\left(\mathrm{C}_{6,22}\right) ; 116.82\left(\mathrm{C}_{8,24}\right) ; 115.47\left(\mathrm{C}_{10,26}\right) ; 90.66$ $\left(\mathrm{C}_{3,19}\right) ; 68.66\left(\mathrm{C}_{12,15}\right) ; 25.38\left(\mathrm{C}_{13,14}\right)$. MS: APCI-HRMS m/z: calculated for $\mathrm{C}_{22} \mathrm{H}_{18} \mathrm{O}_{6}\left(\mathrm{MH}^{+}\right), 379.1176$, found 379.1206 . Yield: $418 \mathrm{mg}, 1.10 \mathrm{mmol}, 35.71 \%$ (211.552\% increase in yield).

\section{BPR 5: 4,4'-[Propane-1,3-diylbis(oxy)]bis(2H-chromen-2-one)}

Employing the above-mentioned procedure (Coumarin-ether dimers (BPR 4-BPR 7)), using 3.08 mmol, $500 \mathrm{mg}, 4 \mathrm{C} ; 6.16 \mathrm{mmol}, 852 \mathrm{mg}, \mathrm{K}_{2} \mathrm{CO}_{3}$ and $3.08 \mathrm{mmol}, 123 \mathrm{mg}$, $\mathrm{NaH}$ (60\% dispersed in oil). Next, $1.54 \mathrm{mmol}, 311 \mathrm{mg}$ 1,3-dibromopropane was slowly added. White crystals formed after recrystallization.

\section{http://repository.uwc.ac.za}


Rf: 0.378. Mp: $203.7-205^{\circ} \mathrm{C} .{ }^{1} \mathrm{H}$ NMR (6oo MHz, CDCI ): $\delta 7.77$ (dd, $J=7.9,1.3 \mathrm{~Hz}, 2 \mathrm{H}, \mathrm{H} 5,18$ ); 7.56-7.48 (m, $2 \mathrm{H}, \mathrm{H}_{7,20}$ ); 7.30 (m, 2H, $\mathrm{H}_{6,19}$ ), 7.25 (dd, $\left.J=10.1,5.0 \mathrm{~Hz}, 2 \mathrm{H}, \mathrm{H}_{8,21}\right) ; 5.72\left(\mathrm{~s}, 2 \mathrm{H}, \mathrm{H}_{3,24}\right) ; 4.38$ (t, $\left.J=5.9 \mathrm{~Hz}, 2 \mathrm{H}, \mathrm{H}_{13,15}\right) ; 2.54\left(\mathrm{~m}, 1 \mathrm{H}, \mathrm{H}_{14}\right) ; 1.63\left(\mathrm{~s}, 2 \mathrm{H}, \mathrm{H}_{2} \mathrm{O} ; \mathrm{CDCI}_{3}\right) .{ }^{13 \mathrm{C} \mathrm{NMR}}\left(151 \mathrm{MHz}, \mathrm{CDCI}_{3}\right): \delta$ $165.22\left(\mathrm{C}_{4,17}\right) ; 162.66\left(\mathrm{C}_{2,23}\right) ; 153.29\left(\mathrm{C}_{9,25}\right) ; 132.62\left(\mathrm{C}_{7,20}\right) ; 124.03\left(\mathrm{C}_{5,18}\right) ; 122.73^{+}\left(\mathrm{C}_{6,19}\right) ; 116.89$ $\left(\mathrm{C}_{8,21}\right) ; 115.37\left(\mathrm{C}_{10,26}\right) ; 90.87\left(\mathrm{C}_{3,24}\right) ; 65.34\left(\mathrm{C}_{13,15}\right) ; 27.95\left(\mathrm{C}_{14}\right)$. MS: APCI-HRMS m/z: calculated for $\mathrm{C}_{21} \mathrm{H}_{16} \mathrm{O}_{6}(\mathrm{MH}), 365.1020$, found 365.1034. Yield: $510 \mathrm{mg}, 1.40 \mathrm{mmol}, 45.45 \%$.

\section{BPR 6: 7,7'-[Propane-1,3-diylbis(oxy)]bis(2H-chromen-2-one)}

Employing the above-mentioned procedure (Coumarin-ether dimers (BPR 4-BPR 7)), using3.08 mmol, $500 \mathrm{mg}, 7 \mathrm{C} ; 6.16 \mathrm{mmol}, 852 \mathrm{mg}, \mathrm{K}_{2} \mathrm{CO}_{3}$ and $3.08 \mathrm{mmol}, 123 \mathrm{mg}$, $\mathrm{NaH}(60 \%$ dispersed in oil) was suspended. Portion-wise, add $1.54 \mathrm{mmol}, 311 \mathrm{mg}$ 1,3-dibromopropane. White crystals formed after recrystallization.

Rf: 0.359. Mp: $177-180^{\circ} \mathrm{C} .{ }^{1} \mathrm{H}$ NMR $(600 \mathrm{MHz}, \mathrm{CDCI}): \delta 7.61(\mathrm{~d}, J=9.5 \mathrm{~Hz}, 2 \mathrm{H}, \mathrm{H}) ; 7.35$ (d, $\left.J=8.6 \mathrm{~Hz}, 2 \mathrm{H}, \mathrm{H}_{5,23}\right) ; 6.88$ (dd, $\left.J=8.6,2.4 \mathrm{~Hz}, 2 \mathrm{H}, \mathrm{H}_{6,24}\right) ; 6.73\left(\mathrm{~s}, 2 \mathrm{H}_{8,18}\right) ; 6.23(\mathrm{~d}, J=9.5 \mathrm{~Hz}$, $\left.2 \mathrm{H}, \mathrm{H}_{3,21}\right) ; 4.21\left(\mathrm{t}, J=6.0 \mathrm{~Hz}, 4 \mathrm{H}, \mathrm{H}_{13,15}\right) ; 2.32\left(\mathrm{~m}, 2 \mathrm{H}, \mathrm{H}_{14}\right) ; 1.64\left(\mathrm{~s}, 2 \mathrm{H}, \mathrm{H}_{2} \mathrm{O} ; \mathrm{CDCI}_{3}\right) .{ }^{13} \mathrm{CNMR}(151 \mathrm{MHz}$, $\left.\mathrm{CDCI}_{3}\right)$ : $\delta 161.89\left(\mathrm{C}_{7,17}\right) ; 161.15\left(\mathrm{C}_{2,20}\right) ; 155.82\left(\mathrm{C}_{9,25}\right) ; 143.36\left(\mathrm{C}_{4,22}\right), 128.80\left(\mathrm{C}_{5,23}\right), 113.19\left(\mathrm{C}_{6,21}\right)$; $112.87\left(\mathrm{C}_{3,24}\right), 112.36\left(\mathrm{C}_{10,26}\right), 101.37\left(\mathrm{C}_{8,18}\right), 64.66\left(\mathrm{C}_{13,15}\right), 28.77\left(\mathrm{C}_{14}\right)$. MS: APCI-HRMS m/z: calculated for $\mathrm{C}_{21} \mathrm{H}_{16} \mathrm{O}_{6}\left(\mathrm{MH}^{+}\right)$, 365.1020, found 365.1042. Yield: $405 \mathrm{mg}, 1.11 \mathrm{mmol}, 36.04 \%$.

\section{BPR 7: 7,7'-[Propane-1,3-diylbis(oxy)]bis(4-methyl-2H-chromen-2-one)}

Employing the above-mentioned procedure (Coumarin-ether dimers (BPR 4-BPR 7)), using 3.41 mmol, $600 \mathrm{mg}$, 4M; $6.82 \mathrm{mmol}, 941 \mathrm{mg}, \mathrm{K}_{2} \mathrm{CO}_{3}$ and $3.08 \mathrm{mmol}, 136 \mathrm{mg}$, NaH (60\% dispersed in oil). To the solution, $1.70 \mathrm{mmol}, 344 \mathrm{mg}$ 1,3-dibromopropane was added, and it wasstirredfor $20 \mathrm{~h}$ at $50^{\circ} \mathrm{C}$. Work up was done using column chromatography DCM:cyclohexane (9:1). White crystals formed after recrystallization.

Rf: 0.208. Mp: $183.5^{-186^{\circ} \mathrm{C} .}{ }^{1} \mathrm{H}$ NMR (6oo MHz, CDCI ): $\delta 7.47$ (d, $\left.J=7.0 \mathrm{~Hz}, 2 \mathrm{H}, \mathrm{H}\right) ; 6.86$ (dd, $J=8.8$. $\left.2.5 \mathrm{~Hz}, 2 \mathrm{H}, \mathrm{H}_{6,22}\right) ; 6.80$ (d, $\left.J=2.5 \mathrm{~Hz}, 2 \mathrm{H}, \mathrm{H}_{8,24}\right) 6.11$ (s, $\left.2 \mathrm{H}, \mathrm{H}_{3,19}\right) ; 4.58\left(\mathrm{~m}, 4 \mathrm{H}, \mathrm{H}_{13,15}\right) ; 5.42$ (dd, $J=$ $\left.17.3,1.4 \mathrm{~Hz}, 2 \mathrm{H}, \mathrm{H}_{14}\right) ; 2.37\left(\mathrm{~s}, 6 \mathrm{H}, \mathrm{H}_{11,27}\right) ; 1.26\left(\mathrm{~s}, 2 \mathrm{H}, \mathrm{H}_{2} \mathrm{O} ; \mathrm{CDCL}_{3}\right) .{ }^{13 \mathrm{C} \mathrm{NMR}}\left(151 \mathrm{MHz}, \mathrm{CDCI}_{3}\right): \delta$ $161.54\left(\mathrm{C}_{2,18}\right) ; 161.29\left(\mathrm{C}_{7,23}\right) ; 155.18\left(\mathrm{C}_{9,25}\right) ; 152.52\left(\mathrm{C}_{4,20}\right) ; 132.15\left(\mathrm{C}_{5,21}\right) ; 125.51\left(\mathrm{C}_{10,26}\right) ; 118.52$ $\left(\mathrm{C}_{6,22}\right) ; 112.78\left(\mathrm{C}_{3,19}\right) ; 112.00\left(\mathrm{C}_{8,24}\right) ; 101.70\left(\mathrm{C}_{13,15}\right) ; 69.20\left(\mathrm{C}_{14}\right) ; 18.67\left(\mathrm{C}_{11,27}\right)$. MS: APCI-HRMS m/z: calculated for $\mathrm{C}_{23} \mathrm{H}_{20} \mathrm{O}_{6}\left(\mathrm{MH}^{+}\right)$, 393.1333, found 391.2808. Yield: $495 \mathrm{mg}, 1.26 \mathrm{mmol}, 36.95 \%$.

\section{Coumarin-thiophene ester conjugate (BPR 8)}

First, activation chemistry was implemented by suspending $3.90 \mathrm{mmol}$, 500mg, 2-thiophenecarboxylic acid (TP) and $5.07 \mathrm{mmol}, 822 \mathrm{mg}, \mathrm{N}, \mathrm{N}^{\prime}$-carbonyldiimidazole (CDI) in $30 \mathrm{~mL}$ dry DCM. The solution was stirred for $2-4 \mathrm{~h}$ at $\mathrm{rt}$ and the solvent removed in vacuo. To the residue obtained, 3.90 
mmol, $632 \mathrm{mg}, 7 \mathrm{C}$ and $7.80 \mathrm{mmol} 1078 \mathrm{mg}, \mathrm{K}_{2} \mathrm{CO}_{3}$ in $40 \mathrm{~mL}$ THF was added and it was stirred for an additional $20 \mathrm{~h}$ at $40^{\circ} \mathrm{C}$. The reaction was cooled down, extracted with $2 \times 60 \mathrm{~mL}$ ethyl acetate and dried using magnesium sulphate. The solution was filtered, dried and purified with column chromatography using DCM:cyclohexane (9:1). It was further concentrated and recrystallized - a white crystalline solid formed.

\section{BPR 8: 2-Oxo-2H-chromen-7-yl-thiophene-2-carboxylate}

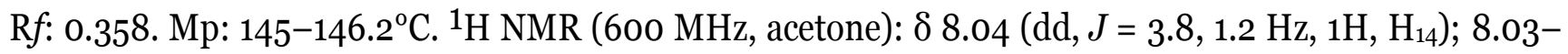
$8.01\left(\mathrm{~m}, 1 \mathrm{H}, \mathrm{H}_{4}\right) ; 8.01\left(\mathrm{~d}, J=1.1 \mathrm{~Hz}, 1 \mathrm{H}, \mathrm{H}_{16}\right) ; 7.78\left(\mathrm{~d}, J=6.6 \mathrm{~Hz}, 1 \mathrm{H}, \mathrm{H}_{5}\right) ; 7.36\left(\mathrm{~m}, 1 \mathrm{H}, \mathrm{H}_{15}\right) ; 7.31(\mathrm{dd}, J=$ 3.4, $2.1 \mathrm{~Hz}, 1 \mathrm{H}, \mathrm{H}_{6}$ ); 7.30 (dd, $\left.J=6.9,1.4 \mathrm{~Hz}, 1 \mathrm{H}, \mathrm{H}_{8}\right) ; 6.42\left(\mathrm{dd}, J=9.6,3.9 \mathrm{~Hz}, 1 \mathrm{H}_{3} \mathrm{H}_{3}\right) ; 2.12-1.96$ (m, 2H, $\mathrm{H}_{2} \mathrm{O}$; acetone). ${ }^{13 \mathrm{C}} \mathrm{NMR}$ (151 MHz, DMSO): $8159.61\left(\mathrm{C}_{2}\right) ; 154.11\left(\mathrm{C}_{7}\right) ; 152.51\left(\mathrm{C}_{12}\right) ; 143.82\left(\mathrm{C}_{9}\right)$; $135.82\left(\mathrm{C}_{4}\right) ; 135.65\left(\mathrm{C}_{13}\right) ; 131.31\left(\mathrm{C}_{16}\right) ; 129.45\left(\mathrm{C}_{5}\right) ; 128.83\left(\mathrm{C}_{15}\right) ; 118.72\left(\mathrm{C}_{6}\right) ; 116.97\left(\mathrm{C}_{10}\right) ; 115.74\left(\mathrm{C}_{3}\right)$; 110.35 $\left(\mathrm{C}_{8}\right)$. MS: APCI-HRMS m/z: calculated for $\mathrm{C}_{16} \mathrm{H}_{18} \mathrm{SO}_{4}\left(\mathrm{MH}^{+}\right)$, 273.0216, found 273.0218. Yield: $402 \mathrm{mg}, 1.48 \mathrm{mmol}, 37.95 \%$.

\section{Coumarin-piperidine ether conjugates (BPR 9, BPR 12, BPR 13)}

In a $100 \mathrm{~mL}$ flask equipped with a cooler, $7.40 \mathrm{mmol}, 1362 \mathrm{mg}$, 1-(2-chloroethyl)-piperidine hydrochloride, $6.17 \mathrm{mmol}, 1000 \mathrm{mg}, 4 \mathrm{C}$ and $12.33 \mathrm{mmol}, 1700 \mathrm{mg}, \mathrm{K}_{2} \mathrm{CO}_{3}$ were suspended in $75 \mathrm{~mL}$ dry acetonitrile. The reaction was refluxed for $10 \mathrm{~h}$ at $80^{\circ} \mathrm{C}$, cooled down, filtered and evaporated under vacuum. The filtrate was then extracted with $100 \mathrm{~mL}$ water and $2 \times 30 \mathrm{~mL}$ ethyl acetate. The combined organic fractions were collected, dried, filtered and concentrated to a volume of $\sim 5 \mathrm{~mL}$. Following recrystallizing at rt, bulky, yellow crystals formed (BPR9).

\section{BPR 9: 4-[2-(Piperidin-1-yl)ethoxy]-2H-chromen-2-one}

Rf: 0.548. Mp: $117-118^{\circ} \mathrm{C} .{ }^{1} \mathrm{H}$ NMR (60o $\left.\mathrm{MHz} \mathrm{CDCI}_{3}\right): \delta 7.78\left(\mathrm{dd}, J=7.9,1.5 \mathrm{~Hz}, 1 \mathrm{H}, \mathrm{H}_{5}\right) ; 7.54-7.50$ $\left(\mathrm{m}, 1 \mathrm{H}, \mathrm{H}_{7}\right) ; 7.29\left(\mathrm{dd}, J=8.3,0.6 \mathrm{~Hz}, 1 \mathrm{H}, \mathrm{H}_{6}\right) ; 7.24\left(\mathrm{~m}, 1 \mathrm{H}, \mathrm{H}_{8}\right) ; 5.66\left(\mathrm{~s}, 1 \mathrm{H}, \mathrm{H}_{3}\right) ; 4.23(\mathrm{t}, J=5.8 \mathrm{~Hz}, 2 \mathrm{H}$, $\left.\mathrm{H}_{12}\right) ; 2.86\left(\mathrm{t}, J=5.8 \mathrm{~Hz}, 2 \mathrm{H}, \mathrm{H}_{13}\right) ; 2.51\left(\mathrm{~s}, 4 \mathrm{H}, \mathrm{H}_{15}, 16\right) ; 1.61-1.55$ (m, 4H, H 17,18$) ; 1.46-1.39$ (m, $2 \mathrm{H}$, $\left.\mathrm{H}_{19}\right) .{ }^{13 \mathrm{CNMR}}\left(151 \mathrm{MHz}, \mathrm{CDCI}_{3}: \delta 165.51\left(\mathrm{C}_{4}\right) ; 162.88\left(\mathrm{C}_{2}\right) ; 153.30\left(\mathrm{C}_{9}\right) ; 132.34\right.$

$\begin{array}{llllll}16,15 & 17,18 & 19 & 16 & 19\end{array}$

$\left(\mathrm{C}_{7}\right) ; 123.84\left(\mathrm{C}_{5}\right) ; 123.04\left(\mathrm{C}_{6}\right) ; 116.74\left(\mathrm{C}_{8}\right) ; 115.69\left(\mathrm{C}_{10}\right) ; 90.57\left(\mathrm{C}_{3}\right) ; 67.70\left(\mathrm{C}_{12}\right) ; 57.01\left(\mathrm{C}_{13}\right) ; 55.01\left(\mathrm{C}_{16,15}\right)$; 25.95 $\left(\mathrm{C}_{17,18}\right)$; 24.01 $\left(\mathrm{C}_{19}\right)$. MS: APCI-HRMS m/z: calculated for $\mathrm{C}_{16} \mathrm{H}_{19} \mathrm{NO}_{3}\left(\mathrm{MH}^{+}\right)$, 274.3270, found 274.1429. Yield: $635 \mathrm{mg}, 2.32 \mathrm{mmol}, 31.35 \%$.

\section{BPR 12: 7-[2-(Piperidin-1-yl)ethoxy]-2H-chromen-2-one}

Employing the above-mentioned procedure (Coumarin-piperidine ether conjugates (BPR 9, BPR 12, BPR 13)), $6.17 \mathrm{mmol}, 1135 \mathrm{mg}$, 1-(2-chloroethyl) piperidine hydrochloride; $6.17 \mathrm{mmol}, 1000 \mathrm{mg}$, $7 \mathrm{C}$ and $12.33 \mathrm{mmol}, 1705 \mathrm{mg} \mathrm{K}_{2} \mathrm{CO}_{3}$. Yellow crystals formed after recrystallization.

Rf: 0.164. Mp: 89.4-90.5 ${ }^{\circ} \mathrm{C} .{ }^{1} \mathrm{H} \operatorname{NMR}\left(600 \mathrm{MHz}, \mathrm{CDCI}_{3}\right): \delta 7.60\left(\mathrm{~d}, J=9.5 \mathrm{~Hz}, 1 \mathrm{H}, \mathrm{H}_{4}\right) ; 7.33(\mathrm{~d}$, $\left.J=8.6 \mathrm{~Hz}, 1 \mathrm{H}, \mathrm{H}_{5}\right) ; 6.82\left(\mathrm{dd}, J=8.6,2.4 \mathrm{~Hz}, 1 \mathrm{H}, \mathrm{H}_{6}\right) ; 6.79$ (d, $\left.J=2.4 \mathrm{~Hz}, 1 \mathrm{H}, \mathrm{H}_{8}\right) ; 6.21(\mathrm{~d}, J=9.5 \mathrm{~Hz}, 1 \mathrm{H}$, $\left.\mathrm{H}_{3}\right) ; 4.12\left(\mathrm{t}, J=6.0 \mathrm{~Hz}, 2 \mathrm{H}, \mathrm{H}_{12}\right) ; 2.76\left(\mathrm{t}, J=6.0 \mathrm{~Hz}, 2 \mathrm{H}, \mathrm{H}_{13}\right) ; 2.47\left(\mathrm{bs}, 4 \mathrm{H}, \mathrm{H}_{15,16}\right) ; 1.60-1.55(\mathrm{~m}, 4 \mathrm{H}$,

\section{http://repository.uwc.ac.za}


$\left.\mathrm{H}_{17,18}\right) ; 1.42\left(\mathrm{bs}, 2 \mathrm{H}, \mathrm{H}_{19}\right) .{ }^{13 \mathrm{C} \mathrm{NMR}}\left(151 \mathrm{MHz}, \mathrm{CDCI}_{3}\right): \delta 162.05\left(\mathrm{C}_{7}\right) ; 161.21\left(\mathrm{C}_{2}\right) ; 155.81\left(\mathrm{C}_{9}\right) ; 143.38$ $\left(\mathrm{C}_{4}\right) ; 128.67\left(\mathrm{C}_{5}\right) ; 113.03\left(\mathrm{C}_{3}\right) ; 112.98\left(\mathrm{C}_{6}\right) ; 112.50\left(\mathrm{C}_{10}\right) ; 101.48\left(\mathrm{C}_{8}\right) ; 66.63\left(\mathrm{C}_{12}\right) ; 57.58\left(\mathrm{C}_{13}\right) ; 55.06$ $\left(\mathrm{C}_{15,16}\right) ; 25.88\left(\mathrm{C}_{17,18}\right) ; 24.09\left(\mathrm{C}_{19}\right)$. MS: APCI-HRMS m/z: calculated for $\mathrm{C}_{16} \mathrm{H}_{19} \mathrm{NO}_{3}\left(\mathrm{MH}^{+}\right)$, 274.1438 , found 274.1434 . Yield: $307 \mathrm{mg}, 1.12 \mathrm{mmol}, 18.15 \%$.

\section{BPR 13: 4-Methyl-7-[2-(Piperidin-1-yl)ethoxy]-2H-chromen-2-one}

Employing the above-mentioned procedure (Coumarin-piperidine ether conjugates (BPR 9, BPR 12, BPR 13)), using $3.41 \mathrm{mmol}, 1600 \mathrm{mg}, 4 \mathrm{M}$ and $6.81 \mathrm{mmol}, 941 \mathrm{mg}, \mathrm{K}_{2} \mathrm{CO}_{3}$. To this solution, 4.09 mmol, $752 \mathrm{mg}$, 1-(2-chloroethyl) piperidine hydrochloride was added. Bulky, yellow crystals formed after recrystallization.

Rf: 0.315. Mp: $102-104^{\circ} \mathrm{C} .{ }^{1} \mathrm{H}$ NMR (6oo MHz, $\left.\mathrm{CDCI}_{3}\right): \delta 7.44\left(\mathrm{~d}, J=8.8 \mathrm{~Hz}, 1 \mathrm{H}, \mathrm{H}_{5}\right), 6.82(\mathrm{dd}$, $\left.J=8.8,2.4 \mathrm{~Hz}, 1 \mathrm{H}, \mathrm{H}_{6}\right), 6.76\left(\mathrm{~d}, J=2.4 \mathrm{~Hz}, 1 \mathrm{H}, \mathrm{H}_{8}\right), 6.08\left(\mathrm{~d}, J=0.9 \mathrm{~Hz}, 1 \mathrm{H}, \mathrm{H}_{3}\right), 4.12(\mathrm{t}, J=5.9 \mathrm{~Hz}, 2 \mathrm{H}$, $\mathrm{H}_{13}$ ), 2.76 (t, $J=5.9 \mathrm{~Hz}, 2 \mathrm{H}, \mathrm{H}_{14}$ ), 2.48 (bs, $\left.4 \mathrm{H}, \mathrm{H}_{16,17}\right), 2.35$ (d, $\left.J=1.0 \mathrm{~Hz}, 3 \mathrm{H}, \mathrm{H}_{11}\right), 1.58(\mathrm{~m}, 4 \mathrm{H}$, $\left.\mathrm{H}_{18,19}\right), 1.41$ (bs, $\left.2 \mathrm{H}, \mathrm{H}_{20}\right)$. ${ }^{13} \mathrm{C} \mathrm{NMR}\left(151 \mathrm{MHz}, \mathrm{CDCI}_{3}\right): \delta 161.77\left(\mathrm{C}_{7}\right) ; 161.23\left(\mathrm{C}_{2}\right) ; 155.13\left(\mathrm{C}_{9}\right) ; 152.48$ $\left(\mathrm{C}_{4}\right), 125.42\left(\mathrm{C}_{5}\right), 113.52\left(\mathrm{C}_{3}\right), 112.56\left(\mathrm{C}_{6}\right), 111.85\left(\mathrm{C}_{10}\right), 101.48\left(\mathrm{C}_{8}\right), 66.44\left(\mathrm{C}_{12}\right), 57.52\left(\mathrm{C}_{13}\right), 55.00$ $\left(\mathrm{C}_{16,17}\right), 25.76\left(\mathrm{C}_{18,19}\right)$, 24.00 $\left(\mathrm{C}_{20}\right), 18.60\left(\mathrm{C}_{11}\right)$. MS: APCI-HRMS m/z: calculated for $\mathrm{C}_{17} \mathrm{H}_{21} \mathrm{NO}_{3}$ $\left(\mathrm{MH}^{+}\right)$, 288.1594, found 288.1593. Yield: $810 \mathrm{mg}, 2.82 \mathrm{mmol}, 82.70 \%$.

\section{Coumarin-morpholine ether conjugates (BPR 10, BPR 11, BPR 14) \\ BPR 10: 4-[2-(Morpholin-4-yl)ethoxy]-2H-chromen-2-one}

Using a $100 \mathrm{~mL}$ flask, $7.40 \mathrm{mmol}, 1378 \mathrm{mg}$, 4-(2-chloroethyl) morpholine hydrochloride; $6.17 \mathrm{mmol}$, $1000 \mathrm{mg}, 4 \mathrm{C}$ and $12.33 \mathrm{mmol}, 1.70 \mathrm{mg}, \mathrm{K}_{2} \mathrm{CO}_{3}$ were combined with a mixture of $75 \mathrm{~mL}$ anhydrous acetonitrile and $5 \mathrm{~mL}$ acetone. The reaction was refluxed at $80^{\circ} \mathrm{C}$ for $5 \mathrm{~h}$, cooled down, filtered and evaporated to dryness. The filtrate was extracted with $100 \mathrm{~mL}$ water and $2 \times 30 \mathrm{~mL}$ ethyl acetate. The organic fractions were combined, dried and filtered. The filtrate was concentrated and recrystallized at $5^{\circ} \mathrm{C}$. Dark, yellowneedles formed(BPR 10).

Rf: 0.622. Mp: $121.3-122^{\circ} \mathrm{C} .1 \mathrm{H}$ NMR (600 MHz, $\left.\mathrm{CDCI}_{3}\right): \delta 7.76$ (dd, $J=7.9,1.5 \mathrm{~Hz}, 1 \mathrm{H}, \mathrm{H} 5$ ); 7.54-7.50 $(\mathrm{m}, 1 \mathrm{H}, \mathrm{H} 7) ; 7.29$ (d, $J=8.3 \mathrm{~Hz}, 1 \mathrm{H}, \mathrm{H} 6) ; 7.27-7.23(\mathrm{~m}, 1 \mathrm{H}, \mathrm{H} 8) ; 5.66(\mathrm{~s}, 1 \mathrm{H}, \mathrm{H} 3) ; 4.24$ (t, $J=5.5 \mathrm{~Hz}, 2 \mathrm{H}$, H12); 3.72-3.69 (m, 4H, H17,18); 2.89 (t, $J=5.5 \mathrm{~Hz}, 2 \mathrm{H}, \mathrm{H} 13) ; 2.60-2.56$ (m, 4H, H15,16). 13CNMR (151 MHz, CDCI3): 8 165.38 (C4); 162.75 (C2); 153.28 (C9); 132.48 (C7); 123.86 (C5); 122.92 (C6); 116.78 (C8); 115.57 (C10); 90.63 ( $\left.\mathrm{C}_{3}\right) ; 67.45$ (C17,18); 66.86 (C12); 56.69 (C13); 53.99 (C15,16). MS: APCI-HRMS m/z: calculated for $\mathrm{C}_{15} \mathrm{H}_{17} \mathrm{NO}_{4}\left(\mathrm{MH}^{+}\right)$, 276.1230, found 278.1229. Yield: $583 \mathrm{mg}, 2.18 \mathrm{mmol}, 29.46 \%$.

\section{BPR 11: 7-[2-(Morpholin-4-yl)ethoxy]-2H-chromen-2-one}

Following the same procedure (Coumarin-morpholine ether conjugates (BPR 10, BPR 11, BPR 14)) as above, using $6.16 \mathrm{mmol}, 1705 \mathrm{mg}$, 4-(2-chloroethyl) morpholine hydrochloride; $6.17 \mathrm{mmol}, 1000$ $\mathrm{mg}, 7 \mathrm{C}$ and $12.33 \mathrm{mmol}, 1170 \mathrm{mg}, \mathrm{K}_{2} \mathrm{CO}_{3}$. Yellow needles formed after recrystallization.

\section{http://repository.uwc.ac.za}


Rf: 0.216. Mp: $118.6-119.1^{\circ} \mathrm{C} .{ }^{1} \mathrm{H} \operatorname{NMR}\left(600 \mathrm{MHz}, \mathrm{CDCl}_{3}\right): \delta 7.61\left(\mathrm{~d}, J=9.5 \mathrm{~Hz}, 1 \mathrm{H}, \mathrm{H}_{4}\right) ; 7.34$ $\left(\mathrm{d}, J=8.6 \mathrm{~Hz}, 1 \mathrm{H}, \mathrm{H}_{5}\right) ; 6.83\left(\mathrm{dd}, J=8.6,2.4 \mathrm{~Hz}, 1 \mathrm{H}, \mathrm{H}_{6}\right) ; 6.79\left(\mathrm{~d}, J=2.4 \mathrm{~Hz}, 1 \mathrm{H}, \mathrm{H}_{8}\right) ; 6.23(\mathrm{~d}, J=9.5 \mathrm{~Hz}$, $\left.1 \mathrm{H}, \mathrm{H}_{3}\right) ; 4.13\left(\mathrm{t}, J=5.6 \mathrm{~Hz}, 2 \mathrm{H}, \mathrm{H}_{12}\right) ; 3.74-3.68\left(\mathrm{~m}, 4 \mathrm{H}, \mathrm{H}_{17,18}\right) ; 2.81\left(\mathrm{t}, J=5.6 \mathrm{~Hz}, 2 \mathrm{H}, \mathrm{H}_{13}\right) ; 2.56(\mathrm{~s}, 4 \mathrm{H}$, $\left.\mathrm{H}_{15,16}\right) .{ }^{13 \mathrm{C} \mathrm{NMR}}\left(151 \mathrm{MHz}, \mathrm{CDCl}_{3}\right): \delta 161.87\left(\mathrm{C}_{7}\right) ; 161.14\left(\mathrm{C}_{2}\right) ; 155.81\left(\mathrm{C}_{9}\right) ; 143.34\left(\mathrm{C}_{4}\right) ; 128.74\left(\mathrm{C}_{5}\right)$; $113.19\left(\mathrm{C}_{10}\right) ; 112.97\left(\mathrm{C}_{6}\right) ; 112.63\left(\mathrm{C}_{3}\right) ; 101.43\left(\mathrm{C}_{8}\right) ; 66.85\left(\mathrm{C}_{17,18}\right) ; 66.40\left(\mathrm{C}_{12}\right) ; 57.28\left(\mathrm{C}_{13}\right) ; 54.07\left(\mathrm{C}_{15,16}\right)$. MS: APCI-HRMS m/z: calculated for $\mathrm{C}_{15} \mathrm{H}_{17} \mathrm{NO}_{4}\left(\mathrm{MH}^{+}\right)$, 276.1230, found 276.1217. Yield: $709 \mathrm{mg}, 2.58$ mmol, $41.88 \%$.

\section{BPR14:4-Methyl-7-[2-(morpholin-4-yl)ethoxy]-2H-chromen-2-one}

Employing the above-mentioned procedure (Coumarin-morpholine ether conjugates (BPR 10, BPR 11, BPR 14)), using $4.09 \mathrm{mmol}, 761 \mathrm{mg}$, 4-(2-chloroethyl) morpholine hydrochloride; $3.41 \mathrm{mmol}$, 600 $\mathrm{mg}, 4 \mathrm{M}$ and $6.81 \mathrm{mmol}, 941 \mathrm{mg}, \mathrm{K}_{2} \mathrm{CO}_{3}$. Yellow needles formed after recrystallization.

Rf: $0.381 . \mathrm{Mp}: 110.4-112.4^{\circ} \mathrm{C} .{ }^{1} \mathrm{H} \operatorname{NMR}\left(600 \mathrm{MHz}, \mathrm{CDCI}_{3}\right): \delta 7.45\left(\mathrm{~d}, J=8.8 \mathrm{~Hz}, 1 \mathrm{H}, \mathrm{H}_{5}\right), 6.82$ (dd, $\left.J=17.4,8.7 \mathrm{~Hz}, 1 \mathrm{H}, \mathrm{H}_{6}\right), 6.76\left(\mathrm{~d}, J=7.5 \mathrm{~Hz}, 1 \mathrm{H}, \mathrm{H}_{8}\right), 6.09$ (d, $J=0.9 \mathrm{~Hz}, 1 \mathrm{H}, \mathrm{H}_{3}$ ), $4.13(\mathrm{t}, J=5.6 \mathrm{~Hz}$, $2 \mathrm{H}, \mathrm{H}_{13}$ ), 3.78-3.60 (m, 4H, H 18,19 ), 2.80 (t, $J=5.6 \mathrm{~Hz}, 2 \mathrm{H}, \mathrm{H}_{14}$ ), 2.56 (m, 4H, H16,17), 2.34(dd, $J=$ 16.2, $\left.0.9 \mathrm{~Hz}, 3 \mathrm{H}, \mathrm{H}_{11}\right)$. ${ }^{13 \mathrm{C}} \mathrm{NMR}\left(151 \mathrm{MHz}, \mathrm{CDCI}_{3}\right)$ : $\delta 161.60\left(\mathrm{C}_{7}\right), 161.20\left(\mathrm{C}_{2}\right), 155.12\left(\mathrm{C}_{9}\right), 152.49\left(\mathrm{C}_{4}\right)$, $125.49\left(\mathrm{C}_{5}\right), 113.64\left(\mathrm{C}_{10}\right), 112.58\left(\mathrm{C}_{6}\right), 111.95\left(\mathrm{C}_{3}\right), 101.40\left(\mathrm{C}_{8}\right), 66.74\left(\mathrm{C}_{13}\right), 66.20\left(\mathrm{C}_{18,19}\right), 57.23\left(\mathrm{C}_{14}\right)$, $53.99\left(\mathrm{C}_{16,17}\right), 18.60\left(\mathrm{C}_{11}\right)$. MS: APCI-HRMS m/z: calculated for $\mathrm{C}_{16} \mathrm{H}_{19} \mathrm{NO}_{4}\left(\mathrm{MH}^{+}\right), 290.1387$, found 290.1380. Yield: $390 \mathrm{mg}, 1.35 \mathrm{mmol}, 39.59 \%$.

\section{Biological evaluation \\ MAO-B inhibition}

A fluorometric assay, based on MAO-B oxidization of kynuramine to 4-hydroxyquinoline, was used to measure the enzyme activities with recombinant human MAO-B (hMAO-B) as enzyme source and kynuramine as substrate [47]. The concentrations of the 4-hydroxyquinoline produced were measured with a fluorescent spectrophotometer (excitation wavelength of $310 \mathrm{~nm}$ and an emission wavelength of $400 \mathrm{~nm}$ ). The fluorescence intensity decreased as 4-hydroxyquinoline production is reduced by the MAO-B test inhibitors. The inhibition potencies were expressed as $\mathrm{IC}_{50}$ values.

\section{Instrumentation, consumables and data processing}

The following were obtained from Sigma Aldrich: Kynuramine.2HBr, microsomes expressed in baculovirus infected Boyce Thompson Institute (BTI) insect cells [48] containing recombinant MAO-B ( $5 \mathrm{mg} / \mathrm{mL}), \mathrm{NaOH}$ and DMSO. A Varian Cary Eclipse ${ }^{\circledR}$ fluorescence spectrophotometer was (Agilent Technologies South Africa, Johannesburg, Gauteng, RSA) utilized for fluorescence spectrophotometry. GraphPad ${ }^{\circledR}$ Prism ${ }^{\circledR}$ V.5.03 software (GraphPad Software, Inc., La Jolla, CA) was utilized for data processing. The non-parametric test application was used for significance comparability.

\section{http://repository.uwc.ac.za}




\section{Methodology}

Recombinant hMAO-B $(5 \mathrm{mg} / \mathrm{mL})$ was stored at $-70^{\circ} \mathrm{C}$. The incubations were prepared in $500 \mu \mathrm{l}$ potassium phosphate buffer $(\mathrm{pH}=7.4)$. Various concentrations of the test inhibitor $(0-100 \mu \mathrm{M})$, in $4 \% \mathrm{DMSO}$ as co-solvent wasprepared. Kynuramine $(30 \mu \mathrm{M})$ served as substrate and was added to each incubation. hMAO-B $(0.0075 \mathrm{mg} / \mathrm{ml})$ was then added and the reactions were incubated for $20 \mathrm{~min}$ at $37^{\circ} \mathrm{C}$. The reaction was terminated by adding $400 \mu \mathrm{L} \mathrm{NaOH}(2 \mathrm{~N})$, and distilled water (1000 $\mu \mathrm{L})$ was added to each reaction. It was centrifuged $(16,000 \mathrm{~g})$ for $10 \mathrm{~min}$ at $\mathrm{rt}$ to produce a supernatant. Concentration measurements of 4-hydroxyquinoline for the incubations were carried out using a Varian Cary Eclipse ${ }^{\circledR}$ fluorescence spectrophotometer (settings: medium photomultiplier tube (PMT) voltage with the excitation and emission slit widths set to $5 \mathrm{~mm}$ ). The supernatant's fluorescence was measured at an excitation wavelength of $310 \mathrm{~nm}$ and an emission wavelength of $400 \mathrm{~nm}$. The initial rate of oxidation was plotted against the logarithm of the inhibitor concentration ([I]) and the $\mathrm{IC}_{50}$ values were determined from the sigmoidal doseresponse curves. Different inhibitor concentrations spanning at least three orders of magnitude were used for each sigmoidal curve $(0 ; 0.1,0.3 ; 1 ; 3 ; 10 ; 30 ; 100 \mu \mathrm{M})$. The inhibition data were fitted to the one site competition model [49] incorporated in the GraphPad ${ }^{\circledR}$ Prism ${ }^{\circledR}$ software package. The $\mathrm{IC}_{50}$ values were determined in triplicate and are expressed as mean \pm standard deviation (SD). As a positive control, we used $(R)$-Deprenyl for its known MAO-B selectivity of 0.020 $\mu \mathrm{M}$ [50] and parent compounds 7-hydroxycoumarin (umbelliferone) because of its known MAO-B inhibitory activity of $28.583 \mu \mathrm{M}$ [51].

\section{AChE-inhibition}

Employing an adapted version of the volumetric method using 5,5'dithiobis(2-nitrobenzoic acid) (DTNB) [52], the test compounds' inhibitory activity against acetylcholinesterase from Electrophorus electricus (electric eel) (eeAChE) was spectrophotometrically evaluated. It is based on the rationale of eeAChE hydrolysis of DTNB, which is measured at $405 \mathrm{~nm}$.

\section{Instrumentation, consumables and data processing}

The following were purchased from Sigma Aldrich: 5,5-dithio-bis-2-nitrobenzoic acid (DTNB), 396.3 $\mathrm{g} / \mathrm{mol})$ ); acetylthiocholine iodide $(289.18 \mathrm{~g} / \mathrm{mol})$; eeAChE (500 UN); albumin from bovine serum (BSA); trizma hydrochloride reagent grade ( $157.60 \mathrm{~g} / \mathrm{mol})$; DMSO and $\mathrm{NaOH}$. The Corning 96-well flat transparent plates were acquired from BioRad (Bio-Rad Laboratories Ltd., Johannesburg, Gauteng, RSA) and a Rayto $6100{ }^{\circledR}$ microplate reader (Rayto Life and Analytical Sciences Co., Ltd., Shenzhen, P.R. China) (filter for $405 \mathrm{~nm}$ ) was utilized. All data analysis and calculations were done

using Prism $4.03{ }^{\circledR}$ (GraphPad ${ }^{\circledR}$, La Jolla, CA). Data analysis was carried out using the Student Newman Keuls multiple range tests and the level of significance was accepted at $p<0.05$. Tacrine $[100 \mathrm{uM}]$ was used as positive control. The data in Table 2 was obtained using equation (1).

$$
\% \text { AChE inhibition }=\frac{\text { Control absorbance }- \text { Test compound absorbance }}{\text { Control absorbance }} \times 100
$$

\section{http://repository.uwc.ac.za}




\section{Methodology}

Stock solutions of test compounds $(1 \mu \mathrm{M}$ and $100 \mu \mathrm{M})$ were prepared by dissolving it in DMSO and it was refrigerated until use. Trizma-hydrochloride buffer was prepared ( $\mathrm{pH}$ adjusted to 8 with diluted $\mathrm{NaOH}$ ) and refrigerated until use. eeAChE (500 UN) was dissolved in $22.727 \mathrm{~mL}$ of Triz-buffer $(38.43 \mathrm{~nm})$, pre-aliquoted $(500 \mu \mathrm{L})$ and frozen. Thus, the final concentration of the aliquots contains $22 \mathrm{UN} / \mathrm{mL}$ in $34.43 \mathrm{mM}$ Tris. Acetylthiocholine iodide ( $15 \mathrm{mM}-5 \mathrm{~mL}, 21.69 \mathrm{mg}$ ), DTNB (1.5 mM-10 mL, 5.94 g) and eeAChE (0.22 units/mL in $50 \mathrm{mM}$ Tris- $\mathrm{HCl}$ and BSA 0.1\%) were prepared directly preceding the assay and protected from light. Continuous thawing and refreezing of the above is not advisable. Then, $148 \mu \mathrm{L}$ DTNB and $50 \mu \mathrm{L}$ of eeAChE solution was added to each of the required wells on the 96-well plate. DMSO $(2 \mu \mathrm{L})$ was added to the control followed by the addition of $2 \mu \mathrm{L}$ of the test compound stock solutions in consecutive wells to give test concentrations of $100 \mu \mathrm{M}$ and $1 \mu \mathrm{M}$. The plate was incubated at $25^{\circ} \mathrm{C}$ for $5 \mathrm{~min}$. Acetylthiocholine iodide $(30 \mu \mathrm{L})$ solution was added to the respective wells by means of a multipipet. Absorbance was measured at $405 \mathrm{~nm}$ every $60 \mathrm{~s}$ for 20 min using the Rayto ${ }^{\circledR} 6100$ microplate reader. The activity (absorbance) was calculated and expressed as a percentage. To obtain a volume of $2000 \mu \mathrm{L}$ of 0.22 units $/ \mathrm{mL}, 50 \mathrm{mM}$ Tris, $0.1 \%$ BSA (sufficient for $40 \times 200 \mu \mathrm{L}$ wells), 1 aliquot of eeAChE was thawed, $20 \mu \mathrm{L}$ of the enzyme was used, and $1980 \mu \mathrm{L}$ Tris $(50.12 \mathrm{nM}-5 \mathrm{~mL}, 39.50 \mathrm{mg})$ and $0.002 \mathrm{~g}$ BSA were added. To compensate for the DMSO effect on activity, the concentration of DMSO was kept at $1 \%$ throughout the assays. This was achieved by the amount of DMSO added to a final volume of 200 $\mu \mathrm{L}$ in the wells, which was maintained at $2 \mu \mathrm{L}(1 \%)$.

\section{Biological results}

The results of the enzyme inhibition activity studies are present in Tables 1 and 2 and Figures 6-8. All the synthesized compounds were included in the biological inhibition studies (Figure 9). 
Table 1. MAO-B inhibition activity of synthesized compounds.

\begin{tabular}{lcc}
\hline Compound & $K_{50}[\mu M]$ & $S D$ \\
\hline (R)-Deprenyl & 0.020 & 0.009 \\
7C & 28.583 & 2.519 \\
4MC & 94.327 & 3.889 \\
BPR 1 & 3.680 & 0.836 \\
BPR 9 & 21.720 & 3.974 \\
BPR 10 & 0.372 & 0.026 \\
BPR 11 & 9.758 & 1.153 \\
BPR 12 & 9.209 & 0.794 \\
BPR 13 & 3.093 & 0.312 \\
BPR 14 & 2.326 & 0.131 \\
\hline
\end{tabular}

Note: Experiments were conducted in triplicate with the mean as $I C_{50}$ values. BPR10 is most active of the test compounds.

Table 2. eeAChE inhibition activity $(100 \mu \mathrm{M}$ and $1 \mu \mathrm{M})$ of the synthesized inhibitors.

\begin{tabular}{lcc}
\hline Compound & \%eeAChE inhibition $[100 \mu M]$ & \%eeAChE inhibition $[1 \mu M]$ \\
\hline Tacrine & $75.81 \pm 1.845^{* * *}$ & $61.52 \pm 5.369^{* * *}$ \\
7 C & IS & $9.585 \pm 2.135$ \\
4M & $11.04 \pm 6.362$ & $8.415 \pm 6.231$ \\
$4 C$ & $11.64 \pm 6.054$ & $12.910 \pm 4.157$ \\
BPR 1 & IS & $13.530 \pm 5.265$ \\
BPR 2 & IS & $22.460 \pm 6.959^{*}$ \\
BPR 3 & IS & $16.450 \pm 8.583$ \\
BPR 4 & IS & $13.870 \pm 4.875$ \\
BPR 5 & IS & $4.760 \pm 7.258$ \\
BPR 6 & IS & $14.960 \pm 4.512$ \\
BPR 7 & IS & $8.910 \pm 3.265$ \\
BPR 8 & IS & $12.090 \pm 3.257$ \\
BPR 9 & $27.77 \pm 8.243^{*}$ & $9.780 \pm 4.268$ \\
BPR 10 & IS & $14.610 \pm 4.568$ \\
BPR 11 & $12.48 \pm 8.075$ & $6.750 \pm 7.239$ \\
BPR 12 & $52.90 \pm 2.621^{* * *}$ & $30.900 \pm 7.259^{* *}$ \\
BPR 13 & $57.43 \pm 2.743^{* * *}$ & $21.560 \pm 5.589^{*}$ \\
BPR 14 & IS & $16.170 \pm 11.250$ \\
\hline
\end{tabular}

Significance: $p<0.05,{ }^{* *} p<0.001,{ }^{* * *} p<0.0001$. All experiments were conducted in triplicate. IS = insoluble. BPR 2, BPR 12, BPR 13 are the most promising of the test compounds.

\section{MAO-Binhibition}

The results of the enzyme inhibition activity studies are presented in Table 1 and Figure 6.

Compounds BPR 2-8 (Figure 9) and 4C (Figure 3) were devoid of MAO-B inhibitory activity. MAO-B inhibition activity was enhanced by nearly one $\log$ unit by conjugation of $7 \mathrm{C}$ (Figures 3 and 6, Table 1; $\mathrm{IC}_{50}=28.583 \mu \mathrm{M}$ ) to erucic acid to yield the coumarin ester, BPR 1 (Figures 6 and 6 and 9, Table 1; $\mathrm{IC}_{50}=3.680 \mu \mathrm{M}$ ), and with the sp carbon 'spacer' to yield coumarin ethers of morpholine BPR 11 (Figures 6 and 9, Table $1 ; \mathrm{IC}_{50}=9.758 \mu \mathrm{M}$ ) and piperidine BPR 12 (Figures 6 and 9, Table $1 ; \mathrm{IC}_{50}=$ 9.209 $\mu \mathrm{M}$ ). Compared to the parent structure, the $4 \mathrm{MC}$ conjugates (Table 1 , Figure $6 ; \mathrm{IC}_{50}=94.327$ $\mu \mathrm{M})$ also revealed a significant increase in activity with the $\mathrm{sp}^{2}$ carbon 'linker' and coumarin ether conjugates of piperidine BPR 13 (Figures 6 and 9, Table $1 ; \mathrm{IC}_{50}=3.093 \mu \mathrm{M}$ ) and morpholine BPR 14 (Figures 6 and 9, Table $1 ; \mathrm{IC}_{50}=2.326 \mu \mathrm{M}$ ). Although $4 \mathrm{C}$ exhibited the lowest inhibition activity, its ether conjugates with piperidine and morpholine, $\mathrm{BPR} 9$ and $\mathrm{BPR} 10$ (Figures 6 and 9 , Table $1 ; \mathrm{IC}_{50}=$ $21.720 \mu \mathrm{M}$ and $0.372 \mu \mathrm{M}$ ) showed activity and produced the most potent compound, BPR10, the 4hydroxycoumarin-morpholine conjugate). 


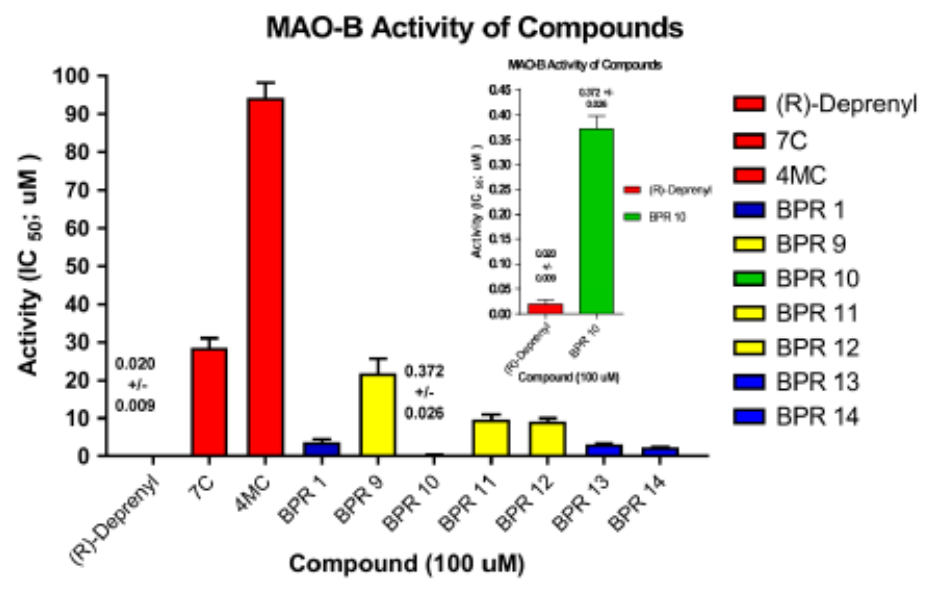

Figure 6. The MAO-B inhibition activity of synthesized inhibitors (Table 1, Figures 1, 3 and 9).

\section{eeAChE inhibition}

The results of the enzyme inhibition activity studies are present in Table 2 and Figures 7 and 8.

The results of the AChE inhibitory activity and structures of the newly synthesized compounds (Figure 9) are presented in Table 2. All of the synthesized compounds revealed weaker AChE potency than the positive control tacrine at both $100 \mu \mathrm{M}$ and $1 \mu \mathrm{M}$.

The coumarin scaffolds used in this study $(7 \mathrm{C}, 4 \mathrm{MC}, 4 \mathrm{C})$ do show some activity but were significantly less than the control tacrine. The conjugated coumarine compounds exhibited more promising activities when compared with the various scaffold.

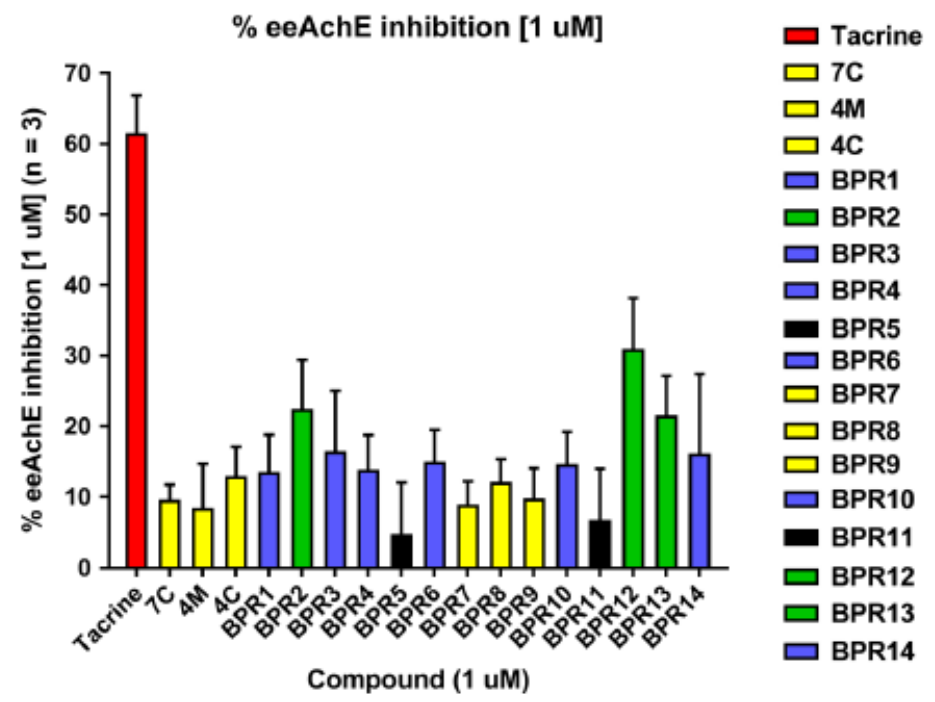

Figure 7. eeAChE inhibition activity $(1 \mu \mathrm{M})$ in the presence of synthesized inhibitors (Table 2, Figures 1, 3 and 9). Tacrine (red bar) represents the positive control. Green bars represent the most potent compounds, followed by compounds with moderate activity (blue bars) of the series. Yellow bars indicate moderate to weak activity while black bars very weak inhibition. 


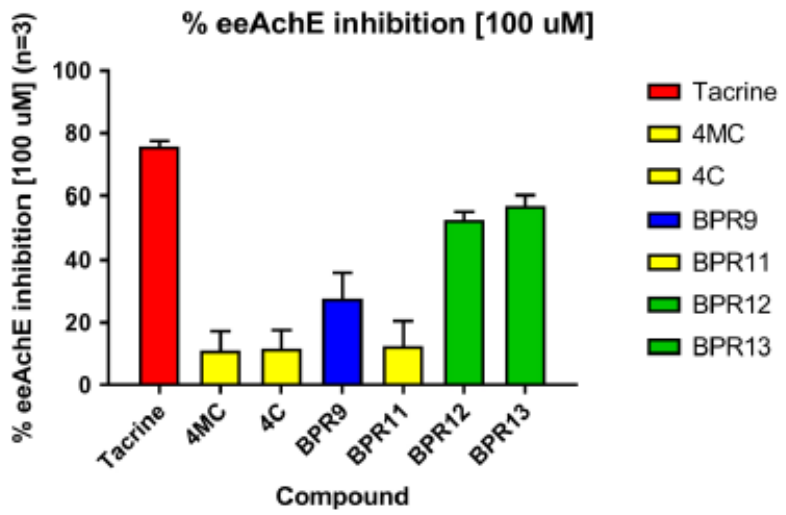

Figure 8. eeAChE inhibition $(100 \mu \mathrm{M})$ activity of synthesized inhibitors (Figures 1, 3 and 9). Tacrine (red bar) represents the positive control. Green bars represent potent activity, blue bars moderate activity while yellow bars weak activity.

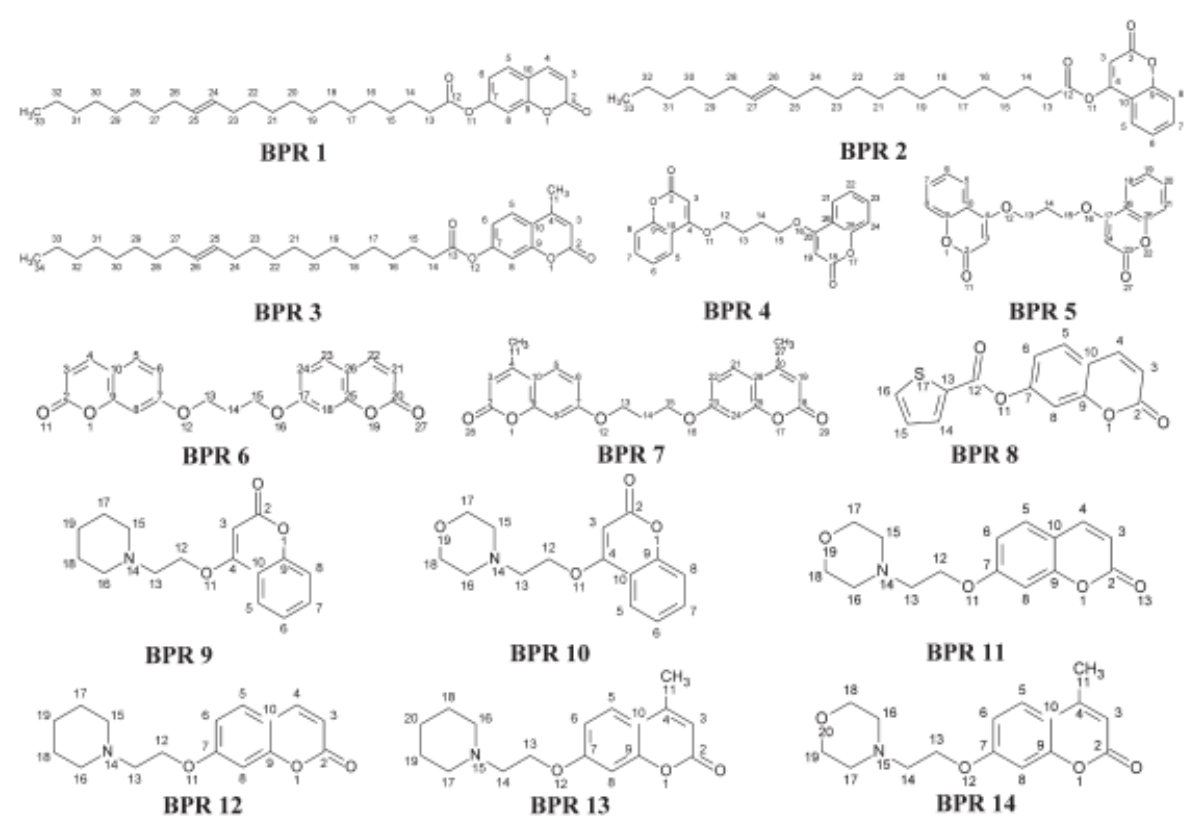

Figure 9. Synthesized compounds used for the relevant enzymatic investigational studies.

The results show that the piperidine compounds, i.e. BPR 12 and BPR 13 (Figures 7, green bar, and 9, Table 2) presented the most promising activity in the AChE inhibition study of $30.90 \%$ and $21.56 \%$, respectively, at $1 \mu \mathrm{M}$. For the coumarin-erucic acid conjugates, BPR 2 (Figures 7, green bar, and 9) showed mild activity at $1 \mu \mathrm{M}$ of $22.460 \%$. Coumarin-morpholine conjugate BPR 10 (Figures 7, blue bar, and 9, Table 2) was less active at $14.61 \%$ inhibition at $1 \mu \mathrm{M}$.

Data from the study at the higher concentration, $100 \mu \mathrm{M}$, revealed eeAChE inhibition of $75.81 \%$ for tacrine (positive control). The piperidine compounds were the most active of all the conjugates at $100 \mu \mathrm{M}$. The piperdines BP R12 and BPR13 (Figures 8, green bar, and 9, Table 2) demonstrated the highest activity in the AChE inhibition study with $52.90 \%$ and $57.43 \%$ inhibition, respectively.

\section{http://repository.uwc.ac.za}


BPR 9 revealed the weakest activity of the piperidine series with $27.77 \%$ at $100 \mu \mathrm{M}$ (Figures 8, blue bar, and 9, Table 2).

The current results demonstrate that compounds BPR 13 (57.43\% at $100 \mu \mathrm{M}$ and $21.56 \%$ at $1 \mu \mathrm{M}$ ), BPR $12(52.90 \%$ at $\mu \mathrm{M}$ and $30.90 \%$ at $1 \mu \mathrm{M})$ and BPR $2(22.46 \%$ at $1 \mu \mathrm{M})$ are the most promising coumarin conjugates (Figures 7-9, Table 2 Graphs 2 and 3 ).

\section{Molecular modelling}

The Material Studio ${ }^{\circledR}$ Discovery Studio ${ }^{\circledR}$ V3.1.1 software package (2012) with the CDOCKER ${ }^{\circledR}$ application was used. The method can be summarized in three parts, namely: preparing the protein, preparing the ligands, and docking and scoring.

\section{Protein preparation}

The appropriate protein crystal structures 4EY7 [22] (crystal structure of recombinant human AChE in complex with donepezil) and 2V61 [20] (crystal structure of human MAO-B in complex with the selective inhibitor 7-(3-chlorobenzyloxy)-4-(methyl amino) methyl coumarin) were downloaded from the Protein Data Bank (www.rcsb/pdb.org). The downloaded files were imported into Discovery Studio ${ }^{\circledR}$ and the protein report was used to confirm and check for any irregularities (crystallographic data, missing regions, alternate conformations, ligand co-crystallized, invalid residues, gaps in chains, etc.). The Prepare Protein ${ }^{\circledR}$ function was used to correct the irregularities mentioned above (when applicable). The co-crystallized ligand was removed and the protein was then typed with CHARMm ${ }^{\circledR}$ (Chemistry at Harvard Macromolecular Mechanics (charmm), Cambridge, MA) forcefield (Partial Charge: Momany and Rone), which assigned partial charges to the structures, and it was then saved. Subsequently, the ionization and protonation state was optimized using 'Protonate only the Protein' and 'Calculate Protein Ionization and Residue pK' with settings: $\mathrm{pH}$ Rang ( $0-14)$. The proteins were minimized (to correct for any potential steric overlap due to the structures' van der Waals interactions). Minimization settings: max steps 5000; implicit solvent model: distance-dependent dielectric for $4 \mathrm{EY}_{7}$ and generalized born with implicit membrane for $2 \mathrm{~V} 61$ was used (since MAO-B is membrane bound). After completion of these modelling setup preparations the structures were saved.

\section{Ligand preparation}

In a new window, the sketching function was used to sketch the ligands. Hydrogens were added, and the geometry 'cleaned' (placing the structures in the correct and optimal conformation regarding their atomical angles). The 'Prepare Ligands' utility was used with settings to true change ionization, generate tautomers and correct for valencies according to the accurate settings, and the file was saved.

\section{Docking}

The prepared protein was opened and typed with CHARMm ${ }^{\circledR}$ force field. The prepared ligand file was recalled. In CDOCKER ${ }^{\circledR}$, the prepared protein and prepared ligand were loaded, and docked with random conformation set to 200 , heating target temperature to $900 \mathrm{~K}$ and use full potential to true.

\section{http://repository.uwc.ac.za}


The best poses (selected according the highest CDOCKER score of the docked ligand within the active site of the protein and the prominent side chains was documented and interpreted (according to Accelrys ${ }^{\circledR}$ Discovery Studio ${ }^{\circledR}$ V3.1.1 protocols, 2012). The CDOCKER ${ }^{\circledR}$ energy is a quantitative indication of the ligand pose best accepted by the protein and includes numerous mathematical models.

\section{Results of the molecular modelling studies MAO-B docking}

The pyrone ring of the coumarin-morpholine conjugate (BPR 10) revealed numerous interactions with pivotal residues in the substrate cavity. Hydrogen binding (distance $=2.015307 \AA$; angle = 109.461123 ${ }^{\circ}$ ) with CYS172 (dotted white line), $\pi$ interaction (distance $=6.514040 \AA$; angle $=$ $22.164892^{\circ}$ ) with TYR398 (orange line) and $\pi$-Sigma interaction (distance $=2.911005 \AA$; angle $=$ 27.035908 ${ }^{\circ}$ ) with LEU171 (orange line) (Figure 10).

The significance of TYR398 and TYR435, which directs a navigation path for the inhibitor towards the flavin adenine dinucleotide (FAD) (Figure 10) is evident. This corresponds with the 'guidance' towards the active site's aromatic cage observation by Akyüz et al. [53].

\section{AChE docking}

BPR 2 and BPR 13 were docked into hAChE to elucidate the binding mode of these novel inhibitors. The interactions with the protein's residues for each compound are described.

Since AChE contains a long narrow gorge that leads towards the catalytic anionic site (CAS) (see below), the elongated fatty acid chain of BPR 2 was preferred, rather than a steric bulky conjugate. Thus, the coumarin entity of BPR 2 occupied the peripheral anionic site (PAS) while the erucic acid enitity was pushed in through the gorge into the CAS (Figures 11 and 12). The rest of the elongated lipophilic entity was influenced by the neighbouring residues and fitted into the gorge to reach the CAS (although no further interactions were observed).

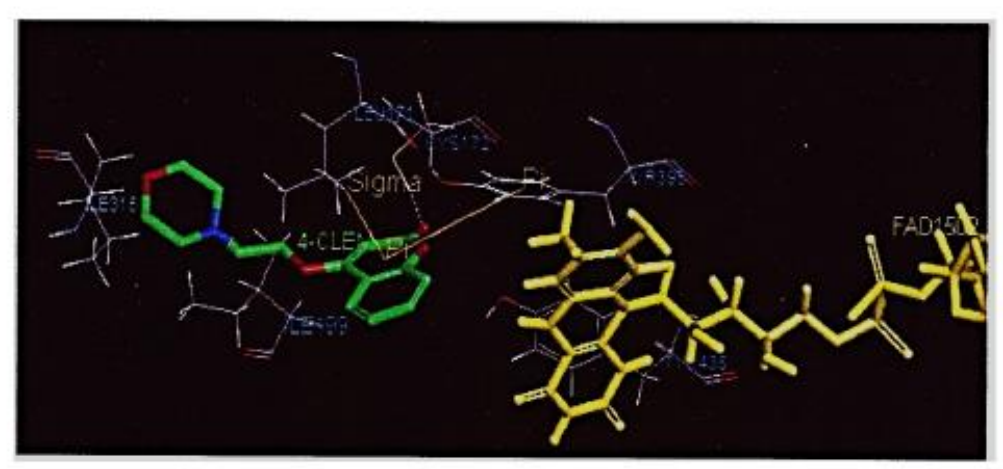

Figure 10. BPR 10 (green ball-and-stick) docked in the hMAO-B protein (2V61). $\pi-\pi$ interactions (orange), Pi-Sigma bindings (orange) and hydrogen binding (white dotted line) are observed in the substrate cavity. 


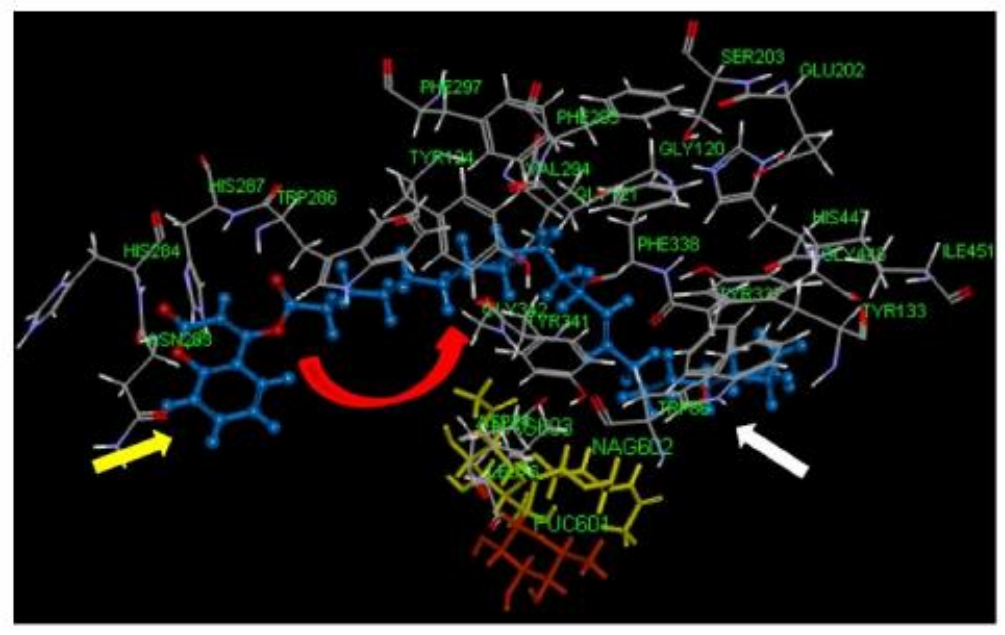

Figure 11. BPR 2 (blue ball-and-stick) docked in the hAChE protein with neighbouring atoms (line models). The coumarin entity (yellow arrow) inside the PAS with the elongated lipophillic entity (red arrow and white arrow) in the gorge and CAS.

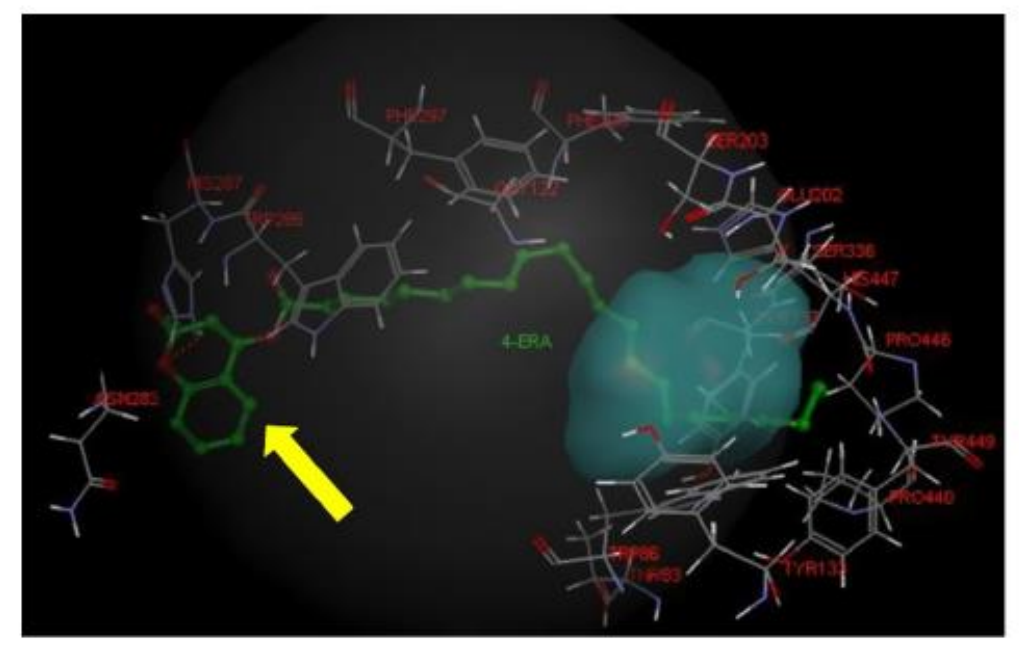

Figure 12. BPR 2 (green ball-and-stick) located in the PAS site of the actives site illustrated in transparent cyan, including the CAS site in transparent blue.

Two hydrogen bonds between the pyrone ring of BPR 2 and HIS287 were observed(Figure 12; yellow arrow; dotted orange and green line) with the lipophillic entity stretching towards the CAS (Figure 12; blue sphere) through the gorge. 


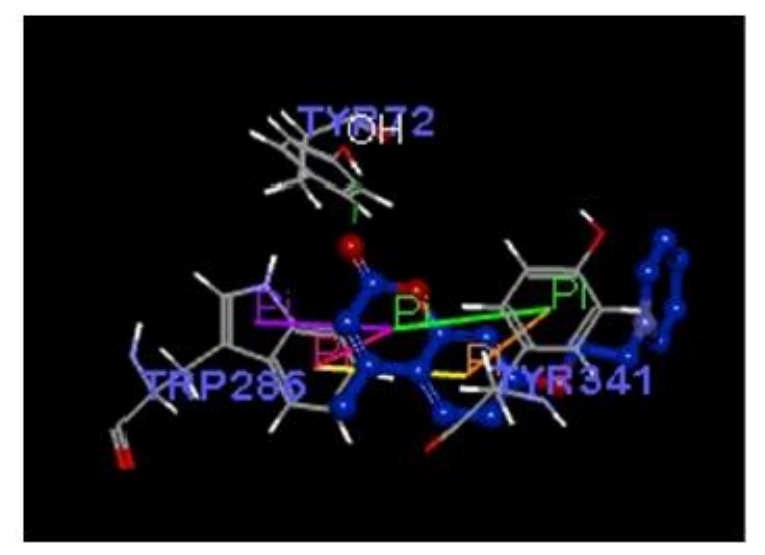

Figure 13. A detailed view of the $5 \pi-\pi$ interactions (violet, pink, yellow, orange and green lines) as well as the $\mathrm{H}$-bond (dotted green line) between the compound BPR 13 and hAChE protein residues.

The molecular modelling investigation of selected coumarin conjugates (BPR 2 and BPR 13 for hACHE and BPR 10 for MAO-B) showed the potential of these conjugates to interact with these enzymes as targets for the treatment of Alzheimer's disease. The aromatic ring scaffold of coumarin has clearly been shown to be an important pharmacophore moiety for $\pi-\pi$ interactions with TYR283 and TRP341 in the PAS region of hAChE. Hydrogen bond formation has also been observed with residual TYR72. Two hydrogen bonds between the pyrone ring of BPR 2 and HIS287 (yellow arrow; dotted orange and green lines) with influential neighbouring atoms (line models) are illustrated for compound BPR 2 (Figure12).

Two $\pi-\pi$ interactions for BPR 13 are shown, the one between TYR341 (Figure 13) and the phenyl ring (orange line) $\left(\right.$ distance $=3.802244 \AA$; angle $=19.229446^{\circ}$ ) and the other between the pyrone ring and $\mathrm{TRP} 341$ (violet line $)\left(\right.$ distance $=4.869986 \AA$; angle $\left.=7.121048^{\circ}\right)$.

\section{Discussion}

The coumarin-morpholine ether conjugate, BPR 10 (4-[2-(morpholin-4-yl)ethoxy]-2Hchromen-2-one) proved to be the most promising hMAO-B inhibitor $\left(\mathrm{IC}_{50}=0.372 \mu \mathrm{M}\right)$. The coumarin-piperidine conjugates BPR 13 (4-methyl-7-[2-(piperidin-1-yl)ethoxy]-2H-chromen-2one) and BPR 12 [(7-[2-(piperidin-1-yl)ethoxy]-2H-chromen-2-one)] were the most potent inhibitors of eeAChE, with an inhibitory activity of $57.43 \%$ at $100 \mu \mathrm{M}$ and $30.90 \%$ at $1 \mu \mathrm{M}$, respectively.

Docking studies revealed that the morpholine-coumarin compound BPR 10 was able to occupy both the entrance and substrate cavities of MAO-B's active site, with the coumarin moiety residing in the substrate cavity, while the morpholine moiety resides in the entrance cavity. BPR 10 shows $\pi-$ interactions with residues of CYS172, LEU171 and ILE198, and a relatively strong H-bond is present between the pyrone ring and CYS172. The coumarin entity of this compound is well positioned in the 'aromatic cage' of the substrate cavity. BPR 13 occupied both the PAS and the CAS of hAChE, with the coumarin positioned in the PAS region, the linker in the gorge (between the PAS and CAS regions) and the piperdine entity in the CAS region. BPR 13 formed $\pi$-interactions with TRP286 and TYR341, and an H-bond withTYR72 in the PAS.

\section{http://repository.uwc.ac.za}


A series of novel coumarin derivatives conjugated to piperidine, morpholine, thiophene and erucic acid structures were successfully synthesized, and biological studies of key enzymes, i.e. MAO-B and $\mathrm{AChE}$ in $\mathrm{AD}$ were studied. Molecular modelling investigations of the selected promising compounds with MAO-B and AChE provided insight into the binding modes and requirements of these structures. The current study showed the coumarin scaffold to be uniquely suitable for designing novel lead structures as MTDLs for AD.

\section{Acknowledgements}

The authors are grateful to the North-West University, University of the Western Cape and the National Research Foundation of South Africa for financial support. Presented at the 9 th International Symposium on Computational Methods in Toxicology and Pharmacology Integrating Internet Resources, CMTPI- 2017, 27-30 October 2017, Goa, India.

\section{Disclosure statement}

No potential conflict of interest was reported by the authors. 


\section{References}

[1] M. Prince, A. Wimo, M. Guerchet, G.-C. Ali, Y.-T. Wu, M. Prina. World Alzheimer report 2015. The global impact of dementia, Alzheimer's Disease International, London, UK. Available at http:// www.worldalzreport2015.org/.

[2] L.E. Hebert, J. Weuve, P.A. Scherr, D.A. Evans. Alzheimer disease in the United States (2010-2050) estimated using the 2010 census, Neurology 7(2013). pp. 1778-1783.

[3] E.K. Perry, R.H. Perry, C.J. Smith, D. Purohit, J. Bonham, D.J. Dick, J.M. Candy, J.A. Edwardson, and A. Fairbairn, Cholinergic receptors in cognitive disorders, Can. J. Neurol. Sci. 13 (1986), pp. 521-527. [4] H.C. Fibiger, Cholinergic mechanisms in learning, memory and dementia: A review of recent evidence, Trends Neurosci. 14 (1991), pp. 220-223.

[5] D. Paterson and A. Nordberg, Neuronal nicotinic receptors in the human brain, Prog. Neurobiol. 61 (2000), pp. 75-111.

[6] E. Perry, C. Martin-Ruiz, M. Lee, M. Griffiths, M. Johnson, M. Piggott, V. Haroutunian, J.D. Buxbaum, J. Nasland, K. Davis, C. Gotti, F. Clementi, S. Tzartos, O. Cohen, H. Soreq, E. Jaros, R. Perry, C. Ballard, I. McKeith, and J. Court, Nicotinic receptor subtypes in human brain ageing, Alzheimer and Lewy body diseases, Eur. J. Pharmacol. 393 (2000), pp. 215-222.

[7] R.T. Mott and C.M. Hulette, Neuropathology of Alzheimer's disease, Neuroimaging Clin. N. Am. 15 (2005), pp. 755-765, ix.

[8] F. Assal and J.L. Cummings, Neuropsychiatric symptoms in the dementias, Curr. Opin. Neurol. 15 (2002), pp. 445-450.

[9] T. Erkinjuntti, Treatment options: The latest evidence with galantamine (Reminyl), J. Neurol. Sci. 203-204 (2002), pp. 125-130.

[10] T. Saito, Y. Takaki, N. Iwata, J. Trojanowski, and T.C. Saido, Alzheimer's disease, neuropeptides, neuropeptidase, and amyloid-beta peptide metabolism, Sci. Aging Knowledge Environ. 2003 (2003), p.Pe1.

[11] P. Kasa, Z. Rakonczay, and K. Gulya, The cholinergic system in Alzheimer's disease, Prog. Neurobiol. 52 (1997), pp. 511-535.

[12] S.K. Mencher and L.G. Wang, Promiscuous drugs compared to selective drugs (promiscuity can be a virtue), BMC Clin. Pharmacol. 5 (2005), p. 3.

[13] F. Li, J.J. Wu, J. Wang, X.L. Yang, P. Cai, Q.H. Liu, L.Y. Kong, and X.B. Wang, Synthesis and pharmacological evaluation of novel chromone derivatives as balanced multifunctional agents against Alzheimer's disease, Bioorg. Med. Chem. 25 (2017), pp. 3815-3826.

[14] S.A. Nisha and K.P. Devi, Gelidiella acerosa protects against Abeta 25-35-induced toxicity and memory impairment in Swiss Albino mice: An in vivo report, Pharm. Biol. 55 (2017), pp. 1423-1435.

[15] P. Riederer, W. Danielczyk, and E. Grunblatt, Monoamine oxidase-B inhibition in Alzheimer's disease, Neurotoxicology 25 (2004), pp. 271-277.

[16] D. Kim, S.H. Baik, S. Kang, S.W. Cho, J. Bae, M.Y. Cha, M.J. Sailor, I. Mook-Jung, and K.H. Ahn, Close correlation of monoamine oxidase activity with progress of Alzheimer's disease in mice, observed by in vivo two-photon imaging, ACS Cent. Sci. 2 (2016), pp. 967-975.

[17] N.T. Tzvetkov and L. Antonov, Subnanomolar indazole-5-carboxamide inhibitors of monoamine oxidase B (MAO-B) continued: Indications of iron binding, experimental evidence for optimised solubility and brain penetration, J. Enzyme Inhib. Med. Chem. 32 (2017), pp. 960-967.

[18] R. Harada, A. Ishiki, H. Kai, N. Sato, K. Furukawa, S. Furumoto, T. Tago, N. Tomita, S. Watanuki, K. Hiraoka, Y. Ishikawa, Y. Funaki, T. Nakamura, T. Yoshikawa, R. Iwata, M. Tashiro, H. Sasano, T. Kitamoto,

\section{http://repository.uwc.ac.za}


K. Yanai, H. Arai, Y. Kudo, and N. Okamura, Correlations of 18F-THK5351 PET with post-mortem burden of tau and astrogliosis in Alzheimer's disease, J. Nucl. Med. 117 (2017), p. 197426.

[19] C. Binda, J. Wang, L. Pisani, C. Caccia, A. Carotti, P. Salvati, D.E. Edmondson, and A. Mattevi, Structures of human monoamine oxidase $B$ complexes with selective noncovalent inhibitors: Safinamide and coumarin analogs, J. Med. Chem. 50 (2007), pp. 5848-5852.

[20] Protein Data Bank, 2 V61 Structure of human MAO B in complex with the selective inhibitor 7(3-chlorobenzyloxy)-4-(methylamino)methyl-coumarin. Available at http://www.rcsb.org/pdb/ explore/explore.do?structureId=2V61.

[21] J. Cheung, M.J. Rudolph, F. Burshteyn, M.S. Cassidy, E.N. Gary, J. Love, M.C. Franklin, and J.J. Height, Structures of human acetylcholinesterase in complex with pharmacologically important ligands, J. Med. Chem. 55 (2012), pp. 10282-10286.

[22] Protein Data Bank, $4 E Y 7$ Crystal structure of recombinant human acetylcholinesterase in complex with Donepezil. Available at http://www.rcsb.org/pdb/explore/explore.do?structureId=4EY7.

[23] N.C. Inestrosa, A. Alvarez, C.A. Perez, R.D. Moreno, M. Vicente, C. Linker, O.I. Casanueva, C. Soto, and J. Garrido, Acetylcholinesterase accelerates assembly of amyloid-beta-peptides into Alzheimer's fibrils: Possible role of the peripheral site of the enzyme, Neuron 16 (1996), pp. 881-891.

[24] C. Garino, T. Tomita, N. Pietrancosta, Y. Laras, R. Rosas, G. Herbette, B. Maigret, G. Quelever, T. Iwatsubo, and J.L. Kraus, Naphthyl and coumarinyl biarylpiperazine derivatives as highly potent human beta-secretase inhibitors. Design, synthesis, and enzymatic BACE-1 and cell assays, J. Med. Chem. 49 (2006), pp. 4275-4285.

[25] L. Yang, Q. Yang, K. Zhang, Y.J. Li, Y.M. Wu, S.B. Liu, L.H. Zheng, and M.G. Zhao, Neuroprotective effects of daphnetin against NMDA receptor-mediated excitotoxicity, Molecules 19 (2014), pp. 1454214555 .

[26] E.C. Pereira, D.L. Lucetti, J.M. Barbosa-Filho, E.M. de Brito, V.S. Monteiro, M.C. Patrocinio, R.R. de Moura, L.K. Leal, D.S. Macedo, F.C. de Sousa, G.S. de Barros Viana, and S.M. Vasconcelos, Coumarin effects on amino acid levels in mice prefrontal cortex and hippocampus, Neurosci. Lett. 454 (2009), pp. 139-142.

[27] A. Hornick, A. Lieb, N.P. Vo, J.M. Rollinger, H. Stuppner, and H. Prast, The coumarin scopoletin potentiates acetylcholine release from synaptosomes, amplifies hippocampal long-term potentiation and ameliorates anticholinergic-and age-impaired memory, Neuroscience 197 (2011), pp. 280-292. [28] D. Oehlrich, F.J. Rombouts, D. Berthelot, F.P. Bischoff, M.A. De Cleyn, L. Jaroskova, G. Macdonald, M. Mercken, M. Surkyn, A.A. Trabanco, G. Tresadern, S. Van Brandt, A.I. Velter, T. Wu, and H.J. Gijsen, Design and synthesis of bicyclic heterocycles as potent gamma-secretase modulators, Bioorg. Med. Chem. Lett. 23 (2013), pp. 4794-4800.

[29] J. Close, R. Heidebrecht Jr, J. Hendrix, C. Li, B. Munoz, L. Surdi, S. Kattar, P. Tempest, P. Moses, X. Geng, B. Hughes, N. Smotrov, C. Moxham, J. Chapnick, I. Kariv, G. Nikov, J.E. Burke, S. Deshmukh, V. Jeliazkova-Mecheva, J.K. Leach, D. Diaz, L. Xu, Z. Yang, G. Kwei, L. Moy, S. Shah, F. Tanga, C. Kenefic,

D. Savage, M. Shearman, R.G. Ball, M.J. McNevin, A. Markarewicz, and T. Miller, Lead optimization of 4,4-biaryl piperidine amides as gamma-secretase inhibitors, Bioorg. Med. Chem. Lett. 22 (2012), pp. 3203-3207.

[30] M.D. McBriar, J.W. Clader, I. Chu, R.A. Del Vecchio, L. Favreau, W.J. Greenlee, L.A. Hyde, A.A. Nomeir, E.M. Parker, D.A. Pissarnitski, L. Song, L. Zhang, and Z. Zhao, Discovery of amide and

\section{http://repository.uwc.ac.za}


heteroaryl isosteres as carbamate replacements in a series of orally active gamma-secretase inhibitors, Bioorg. Med. Chem. Lett. 18 (2008), pp. 215-219.

[31] H. Li, T. Asberom, T.A. Bara, J.W. Clader, W.J. Greenlee, H.B. Josien, M.D. McBriar, A. Nomeir, D.A. Pissarnitski, M. Rajagopalan, R. Xu, Z. Zhao, L. Song, and L. Zhang, Discovery of 2,4,6trisubstituted $N$-arylsulfonyl piperidines as gamma-secretase inhibitors, Bioorg. Med. Chem. Lett. 17 (2007), pp. 6290-6294.

[32] H. Josien, T. Bara, M. Rajagopalan, T. Asberom, J.W. Clader, L. Favreau, W.J. Greenlee, L.A. Hyde, A.A. Nomeir, E.M. Parker, D.A. Pissarnitski, L. Song, G.T. Wong, L. Zhang, Q. Zhang, and Z. Zhao, Small conformationally restricted piperidine $N$-arylsulfonamides as orally active gamma-secretase inhibitors, Bioorg. Med. Chem. Lett. 17 (2007), pp. 5330-5335.

[33] Y.E. Kwon, J.Y. Park, K.T. No, J.H. Shin, S.K. Lee, J.S. Eun, J.H. Yang, T.Y. Shin, D.K. Kim, B.S. Chae, J.Y. Leem, and K.H. Kim, Synthesis, in vitro assay, and molecular modeling of new piperidine derivatives having dual inhibitory potency against acetylcholinesterase and Abeta1-42 aggregation for Alzheimer's disease therapeutics, Bioorg. Med. Chem. 15 (2007), pp. 6596-6607.

[34] H.R. Girisha, J.N. Narendra Sharath Chandra, S. Boppana, M. Malviya, C.T. Sadashiva, and K.S. Rangappa, Active site directed docking studies: Synthesis and pharmacological evaluation of cis-2,6dimethyl piperidine sulfonamides as inhibitors of acetylcholinesterase, Eur. J. Med. Chem. 44 (2009), pp. 4057-4062.

[35] F.C. Meng, F. Mao, W.J. Shan, F. Qin, L. Huang, and X.S. Li, Design, synthesis, and evaluation of indanone derivatives as acetylcholinesterase inhibitors and metal-chelating agents, Bioorg. Med. Chem. Lett. 22 (2012), pp. 4462-4466.

[36] M.R. Jones, E.L. Service, J.R. Thompson, M.C. Wang, I.J. Kimsey, A.S. DeToma, A. Ramamoorthy, M.H. Lim, T. Storr, Dual-function triazole-pyridine derivatives as inhibitors of metal-induced amyloid-beta aggregation, Metallomics 4 (2012), pp. 910-920.

[37] B. Klajnert, T. Wasiak, M. Ionov, M. Fernandez-Villamarin, A. Sousa-Herves, J. Correa, R. Riguera, and E. Fernandez-Megia, Dendrimers reduce toxicity of A 1 1-28 peptide during aggregation and accelerate fibril formation, Nanomedicine 8 (2012), pp. 1372-1378.

[38] M. Malviya, Y.C. Kumar, R.B. Mythri, C. Venkateshappa, M.N. Subhash, and K.S. Rangappa, Muscarinic receptor 1 agonist activity of novel $\mathrm{N}$-aryl carboxamide substituted 3-morpholino arecoline derivatives in Alzheimer's presenile dementia models, Bioorg. Med. Chem. 17 (2009), pp. 5526-5534. [39] Y.C. Kumar, M. Malviya, J.N. Chandra, C.T. Sadashiva, C.S. Kumar, S.B. Prasad, D.S. Prasanna, M.N. Subhash, and K.S. Rangappa, Effect of novel $\mathrm{N}$-aryl sulfonamide substituted 3-morpholino arecoline derivatives as muscarinic receptor 1 agonists in Alzheimer's dementia models, Bioorg. Med. Chem. 16 (2008), pp. 5157-5163.

[40] V. Jeyachandran, R.R. Kumar, M.A. Ali, and T.S. Choon, A one-pot domino synthesis and discovery of highly functionalized dihydrobenzo[b]thiophenes as AChE inhibitors, Bioorg. Med. Chem. Lett. 23 (2013), pp. 2101-2105.

[41] S.B. Bharate, L. Guo, T.E. Reeves, D.M. Cerasoli, and C.M. Thompson, New series of monoquaternary pyridinium oximes: Synthesis and reactivation potency for paraoxon-inhibited electric eel and recombinant human acetylcholinesterase, Bioorg. Med. Chem. Lett. 19 (2009), pp. 5101-5104.

[42] M. Pietsch and M. Gutschow, Synthesis of tricyclic 1,3-oxazin-4-ones and kinetic analysis of cholesterol esterase and acetylcholinesterase inhibition, J. Med. Chem. 48 (2005), pp. 8270-8288.

\section{http://repository.uwc.ac.za}


[43] L. Iuliano, A. Pacelli, M. Ciacciarelli, C. Zerbinati, S. Fagioli, F. Piras, M.D. Orfei, P. Bossu, F. Pazzelli, G. Serviddio, C. Caltagirone, and G. Spalletta, Plasma fatty acid lipidomics in amnestic mild cognitive impairment and Alzheimer's disease, J. Alzheimers Dis. 36 (2013), pp. 545-553.

[44] Y. Okada and M. Okada, Protective effects of plant seed extracts against amyloid beta-induced neurotoxicity in cultured hippocampal neurons, J. Pharm. Bioallied Sci. 5 (2013), pp. 141-147.

[45] R. Cecilia, U. Kunz, and T. Turek, Possibilities of process intensification using microwaves applied to catalytic microreactors, Chem. Engin. Process. Process Intensif. 46 (2007), pp. 870-881.

[46] M. Nuchter, B. Ondruschka, W. Bonrath, and A. Gum, Microwave assisted synthesis - A critical technology overview, Green Chem. 6 (2004), pp. 128-141.

[47] H. Weissbach, T.E. Smith, J.W. Daly, B. Witkop, and S. Udenfriend, A rapid spectrophotometric assay of mono-amine oxidase based on the rate of disappearance of kynuramine, J. Biol. Chem. 235 (1960), pp. 1160-1163.

[48] L. Novaroli, M. Reist, E. Favre, A. Carotti, M. Catto, and P.A. Carrupt, Human recombinant monoamine oxidase B as reliable and efficient enzyme source for inhibitor screening, Bioorg. Med. Chem. 13 (2005), pp. 6212-6217.

[49] L.J. Legoabe, A. Petzer, and J.P. Petzer, alpha-Tetralone derivatives as inhibitors of monoamine oxidase, Bioorg. Med. Chem. Lett. 24 (2014), pp. 2758-2763.

[50] M.J. Matos, C. Teran, Y. Perez-Castillo, E. Uriarte, L. Santana, and D. Vina, Synthesis and study of a series of 3-arylcoumarins as potent and selective monoamine oxidase B inhibitors, J. Med. Chem. 54 (2011), pp. 7127-7137.

[51] S.Y. Kang, K.Y. Lee, S.H. Sung, M.J. Park, and Y.C. Kim, Coumarins isolated from Angelica gigas inhibit acetylcholinesterase: Structure-activity relationships, J. Nat. Prod. 64 (2001), pp. 683-685.

[52] G.L. Ellman, K.D. Courtney, V. Andres Jr, and R.M. Feather-Stone, A new and rapid colorimetric determination of acetylcholinesterase activity, Biochem. Pharmacol. 7 (1961), pp. 88-95. [53] M.A. Akyüz, S.S. Erdem, and D.E. Edmondson, The aromatic cage in the active site of monoamine oxidase B: Effect on the structural and electronic properties of bound benzylamine and $p$ nitrobenzylamine, J. Neural. Transm. (Vienna) 114 (2007), pp. 693-698. 